\title{
Density shocks in the relativistic expansion of highly charged one component plasmas
}

\author{
B. S. Zerbe $\odot^{*}$ and P. M. Duxbury ${ }^{\dagger}$ \\ Department of Physics and Astronomy, Michigan State University, \\ Biomedical \& Physical Sciences Building, 567 Wilson Road East Lansing, Michigan 48824, USA
}

(Received 14 March 2019; published 8 November 2019)

\begin{abstract}
In a previous paper we showed that dynamical density shocks occur in the nonrelativistic expansion of dense single component plasmas relevant to ultrafast electron microscopy; and we showed that fluid models capture these effects accurately. We show that the nonrelativistic decoupling of the relative and center of mass motions ceases to apply and this coupling leads to novel behavior in the relativistic dynamics under planar, cylindrical, and spherical symmetries. In cases where the relative motion of the bunch is relativistic, we show that a dynamical shock emerges even in the case of a uniform bunch with cold initial conditions; and that density shocks are in general enhanced when the relative motion becomes relativistic. Furthermore, we examine the effect of an extraction field on the relativistic dynamics of a planar symmetric bunch.
\end{abstract}

DOI: $10.1103 /$ PhysRevAccelBeams.22.114402

\section{INTRODUCTION}

The expansion dynamics of highly charged plasmas is a fundamental problem in areas ranging from astrophysics to nanotechnology to beam physics. Previous analytic work has focused on initial conditions where a highly charged plasma is cold and has uniform density [1-13]; however, the vast majority of this work, with the exception of Bynchenkov and Kovalev [13], have assumed nonrelativistic conditions. In ultrafast electron microscopy (UEM) and some beam physics applications, electron sources are used to produce dense bunches of charged particles within an intense extraction field that is used to accelerate the distribution to near-luminal speeds. In addition, for sufficient densities, the bunch self-field can result in relativistic velocities within the frame of the bunch. These concerns indicate that a relativistic theory is required in many practical cases, and here we present the relevant theory.

The typical analytic approach to relativistic expansion dynamics of such systems is a treatment based on envelope equations that are predicated on the conservation of emittance and the use of uniform density distributions [2]. Uniform ellipsoidal distributions are particularly amenable to analysis as the self-electrostatic field in these distributions is linear and the expansion dynamics results in a simple

\footnotetext{
*zerbe@msu.edu

duxbury@msu.edu
}

Published by the American Physical Society under the terms of the Creative Commons Attribution 4.0 International license. Further distribution of this work must maintain attribution to the author(s) and the published article's title, journal citation, and DOI. power law growth of the ellipsoid axes-at least in the nonrelativistic regime. Furthermore, it is fairly straightforward to show that uniform ellipsoids conserve emittance as long as the distribution remains uniform and all the particles can be treated as having identical Lorentz factors. Moreover, analysis of beam dynamics such as emittance oscillation [14], emittance compensation [15], and the beam halo [16] generally assume similar uniformlike conditions. However, for electron injectors utilizing photoemission the initial conditions of the bunch is often Gaussian, or at the very least nonuniform, and it has long been known that charge redistribution from the nonuniform to the uniform bunch is one of the major sources of emittance growth [17] suggesting that uniform distributions are at best an idealization that miss much of the physics present in the typical situation.

Numerous works within the UEM literature have already looked at various aspects of the evolution of nonuniform distributions [18-32]. Reed presented a fluid model that described the dynamics of nonuniform bunch expansion under the assumptions that the bunch could be treated as having planar symmetry in the nonrelativistic regime [25]. Nonuniform density evolution of nonrelativistic distributions was extended to the planar, cylindrical, and spherical symmetries by Kovalev and Bynchenkov within the Coulomb explosion literature [33], and our group recently rederived this work within the UEM context [32]. These works show that the expansion dynamics in the planar case differs greatly from symmetries in higher dimensions and verified analytic description of the dynamics utilizing both $\mathrm{N}$-particle and particle-in-cell (PIC) simulations. Within our notation, the analytic descriptions depend on terms that can be written as functions multiplied by the quantity 


$$
D_{01}=\frac{\bar{\rho}_{01}}{\rho_{01}}-1
$$

for planar geometries or by the quantity

$$
D_{0 d}=\frac{\rho_{0 d}}{\bar{\rho}_{0 d}}-1
$$

for cylindrical $(d=2)$ and spherical $(d=3)$ geometries, where $\rho_{0 d}$ is the initial density at location $r_{0}$ and $\bar{\rho}_{0 d}$ is the average density within that location. For the uniform distribution, the local density and the average density are the same so that $D_{0 d}=0$ everywhere and the density evolution's dependence on the aforementioned functions vanishes reducing the dynamics to the uniform dynamics utilized extensively in the literature. Otherwise, these functions play a large role in the density evolution leading to differences in the dynamics of distributions with different initial density profiles. Specifically, both Kovalev and Bychenkov and our group found a density shock, seen in Coulomb explosion studies [5-8,18,25,34], was present in the analytic density evolution of initially Gaussian distributions under both cylindrical and spherical symmetries. We also pointed out that such a shock is absent under planar symmetry unless an appropriate initial chirp in phase space is present.

Both works pointed out that the shock arises as the distribution enters what Kovalev and Bychenkov term the "multiflow" regime. Such a regime can be visualized by considering the phase-space density of a continuous distribution. Initially, the phase density has a nonlinear line of best fit that is one-to-one between position and velocity; this one-to-one relation is preserved while the bunch remains laminar. However, as nonuniform distributions evolve, particles initially more toward the middle of the distribution can overtake outer particles, i.e., crossover. Treating the particles doing the crossover separately from the particles being crossed over, the resulting phase space can be thought of as having multiple expected velocities for the same position, i.e., the distribution is in the multi-flow regime. Kovalev and Bychenkov found that their model correctly captures the density evolution if the portion of the distribution within the multiflow regime is small and the density monotonically decreases from the center [33].

We noted in our previous work that the density evolution seen in PIC simulations using an electromagnetic (EM) solver and relativistic particle pusher, which should capture all relativistic effects if the initial field is accurate, do not significantly differ from our analytic description for the densities analyzed there [32]. Moreover, we have seen that the PIC simulations using an EM solver do not significantly differ from those using an electrostatic (ES) solver with relativistic particle pusher for much higher densities than those examined in our previous work, or in the work described here. Assuming the accuracy of the initial fields, we conclude that the relativistic effects are then adequately captured within the relativistic particle pusher, which is equivalent to simply including the relativistic momentum in the analysis. Precisely such an analysis of the relativistic free-expansion of a spherically-symmetric, cold uniform charge distribution was completed by Bychenkov and Kovalev [13], and part of what we do in this manuscript is extend this analysis to nonuniform cases as well as additional symmetries.

Here we treat charge distributions with general initial spatial distributions starting from rest under planar, cylindrical and spherical geometries introducing a novel length scale that is associated with each symmetry. First in Sec. II A, we present general results applicable to all cases. In Sec. II B we derive expressions for planar symmetry for any arbitrary initial spatial distribution and examine these expressions in the nonrelativistic and highly relativistic limits. We then introduce $M$-shell simulations, which are simulations of $M$ equally charged planes in 1D, and show that these simulations reproduce the density evolution derived analytically. In Secs. II C and II D, we derive relativistic density evolution expressions under cylindrical and spherical symmetries, respectively, for arbitrary initial distributions, and we show that these expressions are consistent with PIC calculations using an EM solver and relativistic particle pusher utilizing the well-known package Warp [35]. Further, we show that $M$-shell simulations, which track $M$ equally charged cylindrical- and sphericalshells in 2- and 3- dimensions, respectively, also capture the same density evolution. We validate these expressions against their nonrelativistic and uniform relativistic counterparts, and we examine the expressions in the highly relativistic limit. In Sec. III we introduce an external extraction field in the case of a planar electron bunch, and we point out that the acceleration from self-fields and external fields do not decouple in the relativistic case. Though the analysis is captured by a straightforward extension of the analysis used for the planar case with no extraction field, the physical effects are quite interesting and relevant to ultrafast electron microscopy. In Sec. IV, we demonstrate how to apply the time dependent distributions to calculate the statistical width of the distribution. Section V contains discussions of the underlying physics of these dynamics; noting in particular the emergence of density shocks due to relativistic effects, even in planar uniform systems where a shock does not emerge in the nonrelativistic limit. Finally, we conclude with Sec. VI.

\section{RELATIVISTIC DENSITY EVOLUTION}

In this section we consider planar, cylindrical and spherical geometries in the case of cold initial conditions and where there is no external electromagnetic field. We treat the dynamics using the relativistic treatment of momentum and energy, however we treat the forces between electrons using electrostatics. We then check the latter approximation under cylindrical and spherical 
geometries using PIC calculations using a full EM solver and find excellent agreement. We start with a general analysis and then specialize in the later three subsections.

\section{A. General considerations}

\section{General formulation}

Consider Lagrangian particles under planar, cylindrical, and spherical symmetries-these particles can be visualized as planes, cylindrical shells, and spherical shells, respectively. Let the position of the particles be parametrized by $z$ in the planar case, $r=\sqrt{x^{2}+y^{2}}$ in the cylindrical case, and $r=\sqrt{x^{2}+y^{2}+z^{2}}$ in the spherical case, which in turn reduces all three situation to similar $1 D$ problems. Further let $y_{d}$ for $d=1,2,3$ represent the appropriate $1 D$ parameter, i.e., $z$ or $r$, for the planar, cylindrical, and spherical symmetric cases. Further, denote $V_{d}$ as the Jacobian of the transformation from the specific symmetry to a $1 D$ formulation, namely $V_{1}=1, V_{2}=2 \pi r$, and $V_{3}=4 \pi r^{2}$. Further, introduce the subscript 0 to indicate the initial (at time 0) value of any parameter; for example, $y_{0,1}$ indicates the initial position of a Langragian particle under the planar model. Likewise, denote $V_{0 d}=V_{d}\left(y_{0 d}\right)$, i.e., $V_{01}=1, V_{02}=2 \pi r_{0}$, and $V_{03}=4 \pi r_{0}^{2}$.

Consider the initial probabilitylike density of particles $\rho_{0, d}$ such that the number density can be expressed as $N \rho_{0, d}$ and the charge density as $e N \rho_{0, d}$. For simplicity in the planar case, assume that $\rho_{01}$ is symmetric about 0 , i.e., $\rho_{01}\left(-z_{0}\right)=\rho_{01}\left(z_{0}\right)$. Further, define the cumulative density function by

$$
P_{0 d}=P_{0 d}\left(y_{0, d}\right)=\int_{0}^{y_{0, d}} \rho_{0, d}\left(y_{d}\right) V_{d} d y_{d}
$$

Notice that $P_{0 d}$ can be physically interpreted as the proportion of the distribution between 0 and $y_{0, d}$, and further that $\lim _{z_{0} \rightarrow \infty} P_{01}\left(z_{0}\right)=\frac{1}{2}$ whereas $\lim _{r_{0} \rightarrow \infty} P_{0 d}\left(r_{0}\right)=$ 1 for the cylindrically and spherically symmetric cases. The initial electric field for each symmetry can be written as

$$
E_{0}\left(y_{0, d}\right)=E_{0 d}=\frac{Q_{\mathrm{tot}, d} P_{0 d}}{V_{0 d} \epsilon_{0}}=E_{T d} P_{0 d}
$$

where $Q_{\mathrm{tot}, d}$ is the charge density with units of charge per area, charge per length, and charge in the planar, cylindrically, and spherically symmetric cases, respectively, and $E_{T d}=\frac{Q_{\text {tot, } d}}{V_{0 d} \varepsilon_{0}}$.

To make analytic progress we make the laminar fluid approximation, which states that there is no mixing of the charged particle trajectories. As a result, the symmetries of the charge distributions are conserved. If we consider a particle of charge $q$ and rest mass $m$ starting from rest (cold initial conditions); at position $y_{0, d}$, we want to find the position and velocity of the particle at later times. In a planar system we thus want to find $z\left(z_{0}, t\right)=z$ and $v\left(z_{0}, t\right)=v$; while in the cylindrical and spherical cases we want to find the radial position and radial velocity $r\left(r_{0}, t\right)=r ; v\left(r_{0}, t\right)=v$. One approach is to use the relativistic form of Newton's second law,

$$
\frac{d p}{d t}=q E
$$

where we are considering the $z$ component of these vectors in planar geometries and the radial component in cylindrical and spherical geometries. We use the relativistic expression for the momentum,

$$
p=\gamma m v
$$

where $m$ is the rest mass and

$$
\gamma=\frac{1}{\sqrt{1-\left(\frac{v}{c}\right)^{2}}} .
$$

Due to the laminar fluid property, the electric field experienced by a particle at position $y_{d}\left(y_{0, d}, t\right)$ can be related to the electric field this particle experienced at its initial position, so that

$$
E_{d}\left(y_{d}\right)=\frac{y_{0, d}^{d-1}}{y_{d}^{d-1}} E_{0 d}
$$

These expressions may be used with Newton's second law to solve for the particle dynamics. Alternatively in an energy formulation, conservation of energy requires that the change in kinetic energy equals the change in potential energy. We use the relativistic form of the kinetic energy,

$$
K=(\gamma-1) m c^{2} .
$$

The change in potential energy is found by integrating the force, $F=q E$, in Eq. (8), and the resulting kinetic energy in the planar case,

$$
K_{1}\left(z_{0} ; t\right)=q E_{01}\left(z(t)-z_{0}\right),
$$

the cylindrical case,

$$
K_{2}\left(r_{0} ; t\right)=q E_{02} r_{0} \ln \left(\frac{r(t)}{r_{0}}\right),
$$

and for the spherical case,

$$
K_{3}\left(r_{0} ; t\right)=q E_{03} r_{0}^{2}\left(\frac{1}{r_{0}}-\frac{1}{r(t)}\right) .
$$

From Eqs. (9)-(12) a general relation between the velocity and position is found to be, 


$$
v=\frac{\sqrt{\left(1-\frac{K}{m c^{2}}\right)^{2}-1}}{1-\frac{U}{m c^{2}}} c .
$$

Moreover, since $K$ only depends explicitly on the position, this equation may be integrated to find an expression relating time and position,

$$
t-t_{0}=\int_{y_{0}}^{y} \frac{1}{v} d y .
$$

To obtain an expression for the time evolution of the density, we use the conservation of the charge density under laminar conditions. This conservation can be stated as

$$
\rho_{0 d} V_{d}\left(y_{0}\right) d y_{0}=\rho_{d}(y ; t) V_{d}(y) d y
$$

where $d$ is 1,2 , and 3 for the planar, cylindrical, and spherical symmetry, respectively, and again using $y=$ $\{z, r, r\}$ for planar, cylindrical, and spherical symmetric cases, respectively. In general, this results in the relationship between the density and the initial density of

$$
\rho_{d}(y ; t)=\frac{\rho_{0 d}}{\left(\frac{y}{y_{0}}\right)^{d-1} y^{\prime}},
$$

where ${ }^{\prime} \equiv \frac{d}{d y_{0}}$ with the $d$ 's in this last expression representing differentiation-not dimensionality of the problem.

The original spatial density, $\rho_{0 d}\left(y_{0}\right)$, can also be used to obtain an energy density, $\nu_{d}\left(K_{d} ; t\right)$, by changing the variable from $y_{0}$ to $K_{d}$ through the use of Eqs. (10)-(12). Namely, the energy density function is given by

$$
\nu_{d}\left(K_{d}, t\right)=\sum_{y_{0, d} \in \mathcal{R}} \frac{V_{0 d} \rho_{0, d}\left(y_{0, d}\right)}{\left|\frac{d K_{d}}{d y_{0, d}}\right|}
$$

where $\mathcal{R}$ represents the set of values of $y_{0, d}$ that give the kinetic energy $K_{d}$ at time $t$. Notice that in the planar case, $\mathcal{R}$ always contains two values, $z_{0}$ and $-z_{0}$, regardless of the distribution; however, in the cylindrical and spherical cases, $\mathcal{R}$ does depend on the distribution. Conducting the differential in Eq. (17) we obtain

$$
\nu_{1}\left(K_{1} ; t\right)=\frac{1}{\left|q E_{T 1} z_{0}\right|} \frac{1}{\left|\frac{K_{1}}{q E_{T 1} P_{01} z_{0}}+\frac{\bar{\rho}_{01}}{\rho_{01}}\left(z^{\prime}-1\right)\right|}
$$

and

$$
\nu_{d}\left(K_{d} ; t\right)=\sum_{r_{0} \in \mathcal{R}} \frac{1}{\left|q E_{T d} r_{0}\right|} \frac{1}{\left|\frac{K_{d}}{q E_{T d} P_{0 d} r_{0}}+\frac{1}{d} \bar{\rho}_{0 d}\left(\left(\frac{r_{0}}{\rho_{0 d}}\right)^{d-1} r^{\prime}-1\right)\right|}
$$

where $d$ represents only 2 or 3 in the second equation. Notice the similarity between Eqs. (18) and (19); however, also notice that we did the sum in Eq. (17) for the planar case with the knowledge that every $z_{0}$ appears either with a positive or a negative sign only. This assumption changes when the bunch is in the presence of an acceleration field, which we explicitly show in a later section.

\section{Fundamental parameters}

Some fundamental parameters need to be considered in the discussion of high density single component plasmas. The first is the plasma frequency,

$$
\omega_{p}=\sqrt{\frac{q^{2} n}{\epsilon_{0} m}}
$$

which describes the frequency of coherent plasma oscillations, where $n$ is the number density (number of particles per unit volume) and $q$ the particle charge. We note that relativistic effects affect the plasma frequency, but as our distribution is starting from rest, it is sufficient to consider the nonrelativistic plasma frequency; however, the plasma frequency for different symmetries is not apparent from Eq. (20). We define average initial densities $\bar{\rho}_{01}=\frac{P_{01}}{z_{0}}$, $\bar{\rho}_{02}=\frac{P_{02}}{\pi r_{0}^{2}}, \bar{\rho}_{03}=\frac{P_{03}}{\frac{4}{3} \pi r_{0}^{3}}$ which are the average densities inside distance $z_{0}$ (planar case), or inside radius $r_{0}$ for the cylindrical and spherical cases. These definitions are used to define initial plasma frequencies as

$$
\omega_{0 d}=\sqrt{\frac{q Q_{\mathrm{tot}, d} \bar{\rho}_{0 d}}{\epsilon_{0} m}}=\sqrt{\frac{d q E_{0 d}}{m y_{0, d}}},
$$

for $d \in\{1,2,3\}$ for the planar, cylindrical, and spherical symmetric cases, respectively.

As will be seen below, the time $\tau_{0 d}$ defined as,

$$
\tau_{0 d}=\frac{2 \pi}{\omega_{0 d}}
$$

sets the timescale for the relativistic expansion of high density charge clouds; as was found in the nonrelativistic cases [32].

In addition to the plasma frequency, we find it advantageous to define the related 1D-probability-like density as

$$
\rho_{r 0 d}=\frac{q E_{0 d}}{m c^{2}}=\frac{q E_{T d} P_{0 d}}{m c^{2}}
$$

where $\rho_{r 0 d}$ has units of inverse length. We call $\rho_{r 0 d}$ the relativistic crossover density for planar, cylindrical, and spherical symmetries for $d=1,2,3$, respectively. The relativistic crossover density is related to the plasma frequency through 


$$
\rho_{r 0 d}=\frac{y_{0, d}}{d} \frac{\omega_{0 d}^{2}}{c^{2}}
$$

The physical interpretation of the relativistic crossover density is that it provides a scale for the kinetic energy as $\frac{K}{m c^{2}}=\rho_{r 01}\left(z-z_{0}\right), \frac{K}{m c^{2}}=\rho_{r 02} r_{0} \ln \left(\frac{r}{r_{0}}\right)$, and $\frac{K}{m c^{2}}=$ $\rho_{r 03} r_{0}\left(1-\frac{r_{0}}{r}\right)$ for the planar, cylindrical, and spherical symmetric cases, respectively. The relativistic length scale, $l_{r 0 d}$ is related to the relativistic density through,

$$
\begin{aligned}
l_{r 0 d} & =\frac{P_{0 d}}{\rho_{r 0 d}} \\
& =\frac{m c^{2}}{q E_{T d}}
\end{aligned}
$$

where $l_{r 0 d}$ is seen to be independent of the initial distribution. $l_{r 0 d}$ can be thought of as the distance a particle experiencing the force obtained by the full distribution at the given coordinate needs to travel before having kinetic energy of $m c^{2}$. Notice, that $l_{r 01}$ is a constant and is specifically independent of $z_{0}$; however, $l_{r 02} \propto r_{0}$ and $l_{r 01} \propto r_{0}^{2}$.

\section{B. Planar symmetry}

In this case, Eq. (13) becomes

$$
v=\frac{\sqrt{\left(1+\rho_{r 01}\left(z-z_{0}\right)\right)^{2}-1}}{1+\rho_{r 01}\left(z-z_{0}\right)} c
$$

where $\rho_{r 01}$ is from Eq. (23). The integral in Eq. (14) may be carried out to find,

$$
t=\frac{\sqrt{\left(1+\rho_{r 01}\left(z-z_{0}\right)\right)^{2}-1}}{\rho_{r 01} c},
$$

which can be inverted to find $z\left(z_{0}, t\right)$ as

$$
z=z_{0}+\frac{1}{\rho_{r 01}}\left(f_{1}\left(z_{0}, t\right)-1\right)
$$

where

$$
f_{1}\left(z_{0}, t\right)=\sqrt{1+\left(\rho_{r 01} c t\right)^{2}}
$$

Taking the time derivative of Eq. (28), the velocity as a function of time becomes,

$$
v=\frac{\rho_{r 01} c t}{f_{1}\left(z_{0}, t\right)} c
$$

From Eq. (16), we find the density dynamics,

$$
\rho_{1}(z ; t)=\frac{\rho_{01}}{1+\frac{d \rho_{r 01}}{d z_{0}}\left[\frac{(c t)^{2}}{f_{1}\left(z_{0}, t\right)}-\frac{\left(f_{1}\left(z_{0}, t\right)-1\right)}{\rho_{r 01}^{2}}\right]}
$$

where

$$
\frac{d \rho_{r 01}}{d z_{0}}=\frac{q Q_{\mathrm{tot}, 1} \rho_{01}}{\epsilon_{0} m c^{2}}=\frac{\rho_{01}}{l_{r 01}}=\frac{\rho_{01}}{\bar{\rho}_{01}} \frac{\omega_{01}^{2}}{c^{2}} .
$$

The corresponding expression for the energy density function from Eq. (18) is

$$
\nu_{1}\left(K_{1} ; t\right)=\frac{l_{r 01}}{2 m c^{2}} \frac{f_{1}\left(z_{0}, t\right)}{\rho_{r 01} c^{2} t^{2}} .
$$

The planar energy distribution can be shown to be independent of the initial spatial distribution by writing it in terms of $v$, which is a function of the force which in turn is a function of particle order only. Specifically,

$$
\nu_{1}\left(K_{1} ; t\right)=\frac{1}{2 m c^{2}} \frac{l_{r 01}}{v t} .
$$

In turn, this can be expressed as a function of $K_{1}$ using Eq. (26) as

$$
\nu_{1}\left(K_{1} ; t\right)=\frac{1}{2 m c^{2}} \frac{l_{r 01}}{c t} \frac{1+\frac{K_{1}}{m c^{2}}}{\sqrt{\left(1+\frac{K_{1}}{m c^{2}}\right)^{2}-1}} .
$$

The nonrelativistic limit occurs when $\rho_{r 01} c t \ll 1$ or equivalently when $t \ll t_{x}$ where

$$
t_{x}=\frac{1}{\rho_{r 01} c},
$$

and in this limit the expressions above reduce to the known density results, i.e., $z=z_{0}+q E_{01} t^{2} / 2 m$ and

$$
\rho_{1 N R}(z ; t)=\frac{\rho_{01}}{1+\frac{q Q_{\text {tot } 1} \rho_{01} t^{2}}{2 \epsilon_{0} m}}=\frac{\rho_{0}}{1+\frac{1}{2} \frac{\rho_{0}}{\bar{\rho}_{01}}\left(\omega_{01} t\right)^{2}}
$$

where $\rho_{1 N R}(z ; t)$ is the density in the nonrelativistic limit and $\omega_{01}$ is the plasma frequency defined in Eq. (21) $[25,32]$.

The highly relativistic limit is when $\rho_{r 01} c t \gg 1$ or equivalently $t \gg t_{x}$. Note that this second inequality implies that any point in the distribution except the center point at $z_{0}=0$ becomes highly relativistic for sufficient time; this is part of the nature of the planar symmetry, and we find similar nature for the cylindrical symmetry below. In this limit, we find,

$$
z \rightarrow z_{0} \pm c t
$$

where the sign of the luminal velocity is determined by on which side of $z_{0}=0$ the particle originated and 


$$
\rho_{1 H R}(z ; t) \rightarrow \frac{\rho_{01} \rho_{r 01}}{\rho_{r 01}+\frac{1}{l_{r 01}} \rho_{r 01}},
$$

where $\rho_{1 H R}(z ; t)$ is the density distribution in the highly relativistic limit. As there is no crossover in this situation, there is no multi-flow regime in the planar case and this distribution is exact. The interpretation of this result is interesting. First, the majority of the distribution essentially becomes two pulses traveling at near luminal speeds away from one another. Second, as the particles within the distribution reach luminal speeds, the density no longer significantly changes as the particles propagate to the left or right; that is, the density evolves toward an "asymptotic density" determined by Eq. (39). If $\rho_{r 01} \ll \rho_{01}$, then $\rho_{1 H R} \rightarrow P_{01} \rho_{r 01}$; however, if $\rho_{01} \gg \rho_{r 01}$, then on the edges $\rho_{H R} \rightarrow \rho_{01}$ whereas as you go further in the distribution transitions to $P_{01} \rho_{r 01}$. Additionally, the ion energy distribution functions' limits under these conditions are $\frac{1}{m c^{2}} P_{01}$ and $\frac{1}{m c^{2}} \frac{\rho_{01}}{\rho_{r 01}}$. This behavior for the uniform and Gaussian distributions for various ratios of $\frac{l_{r 01}}{L_{0}}$, where $L_{0}$ indicates the original width, may be seen in Fig. 1.

Analytically, for the case of an initial uniform distribution, $\rho_{01}=\frac{1}{L_{0}}$ and $P_{0}=\frac{2 z_{0}}{L_{0}}=2 z_{0} \rho_{01}$ where $L_{0}$ is the initial width of the distribution. In this case,

$$
\rho_{1 H R}(z) \rightarrow \frac{\left(2 z_{0}\right)^{2}}{\left(2 z_{0}\right)^{2} L_{0}+l_{r 01} L_{0}^{2}} .
$$

Thus, the shape of this asymptotic distribution is determined entirely by the length scale, $l_{r 01}$, and the initial width, $L_{0}$. For any point $z_{0} \ll l_{r 01}$, including the entire distribution if $L_{0} \ll l_{r 01}$, this asymptotic density is essentially parabolic with zero density at the center and $\frac{1}{l_{r 01}}$ at the edge. This case can be seen in Fig. 3(a). For extremely dense distributions where $L_{0} \gg l_{r 01}$, the asymptotic density at the edges approaches the original density, $\rho_{01}$. There is also a period of transition between the parabolic and original density when the length scale is much smaller than the original width. Both asymptotic behaviors can be seen in Fig. 1 for both the uniform and Gaussian cases.

The mechanism for the relativistic peak emergence may be further seen in Fig. 2 where the density associated with different Lagrangian particles within the distribution are tracked and shown to asymptote to various density values predicted by Eq. (39). One way to describe this mechanism is to notice that all particles, excepting the center particle, in a planar model will asymptote to the speed of light. As the density is physically smooth, the particles' velocities in the neighborhood of the Lagrangian particle asymptote similarly to the speed of light. In other words, the relative velocity of the particles in the Lagrangian particle's neighborhood asymptotes to zero, and the particles cease to spread in the $z$ dimension. As the $z$ dimension is the only dimension in which the density is spreading in the planar model, this is the same as freezing the density to a constant value-an asymptote. Moreover, as particles toward the edge of the distribution have larger accelerations, these particles asymptote earlier than particles farther in. These differences in "freezing" time result in the middle of the distribution expanding, and becoming less dense, before the onset of the relativistic regime. Coupled with the initial

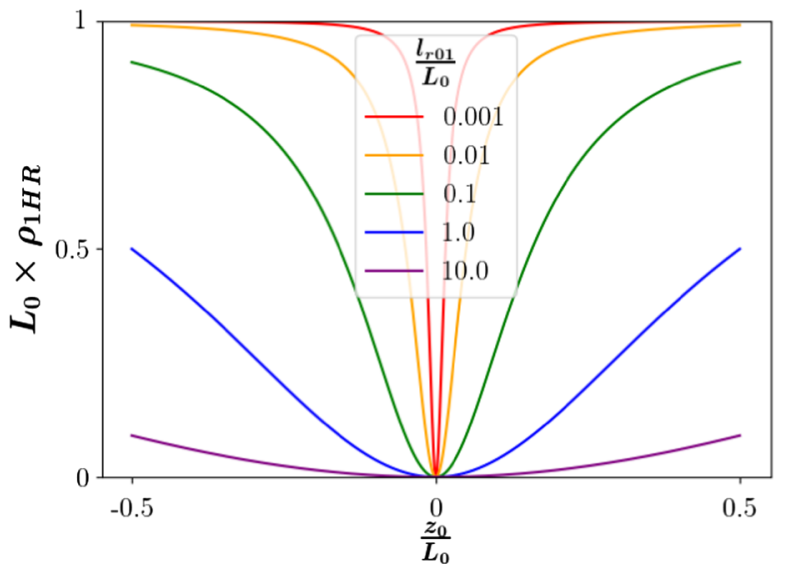

(a)

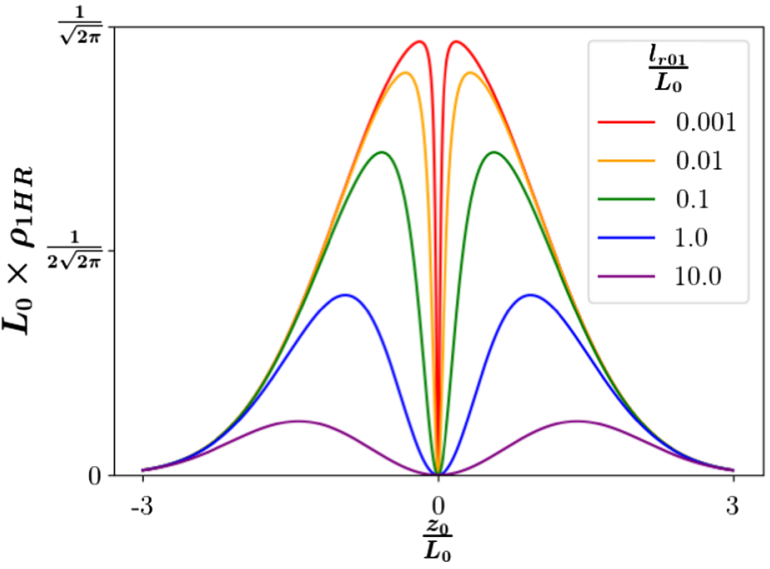

(b)

FIG. 1. Shape of the planar symmetric asymptotic density for (a) uniform and (b) Gaussian initial distributions. $L_{0}$ represents the initial width of the distribution, $l_{r 01}=\frac{\epsilon_{0} m c^{2}}{q Q_{\mathrm{tot}, 1}}$ is the length scale associated with the density of the particles, and $Q_{\mathrm{tot}, 1}$ is the charge per unit area of the distribution as described in the text. Notice that these graphs are independent of the exact choices of $L_{0}$. Further notice the quadratic like behavior in the middle as well as at large values of $\frac{l_{r 01}}{L_{0}}$ for the uniform distribution. Finally note the fact that the distribution approaches the original distribution at its maximum value when $\frac{l_{r 01}}{L_{0}}$ is small. What is not displayed is that the maximum peak is proportional to $\frac{1}{l_{r 01}}$ when $\frac{l_{r 01}}{L_{0}}$ is large. 


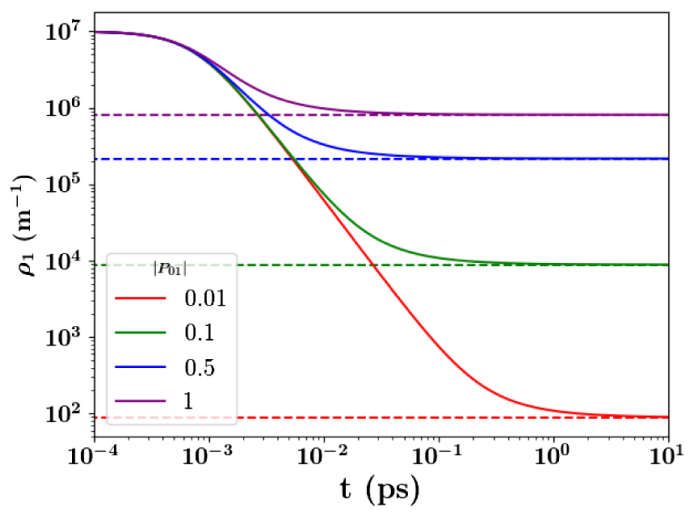

(a) uniform

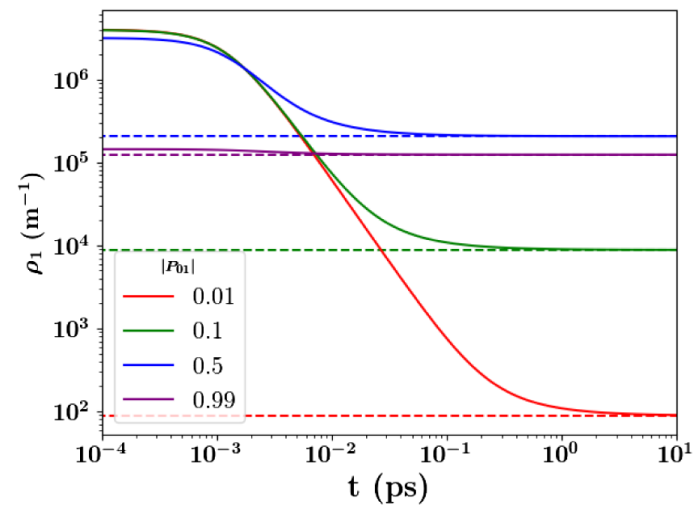

(b) Gaussian

FIG. 2. Theoretical density evolution (solid lines) of specific distribution points in the free expansion of the bunch demonstrating the origin of the relativistic shock. The dotted lines indicate the asymptotic value determined by Eq. (39). The points correspond to locations that symmetrically contain approximately 1\%, 10\%, 50\%, and either 100\% (uniform) or 99\% (Gaussian) of the distribution as indicated by their $P_{01}$ value. The timescales of all points inversely correlate with their location in the distribution [see Eq. (36)]. For the uniform distribution (a), all points start at the same density but converge to different asymptotes according to the inverse relationship between position and timescale. This leads to a parabolic distribution as seen in Eq. (40) as $L_{0} \ll l_{r 01}$ here. For the Gaussian distribution (b), the fact that the outer points have lower initial densities leads to density trajectories crossing indicating the formation of the density peak that goes both up and down in contrast to the sharp peak seen in the uniform case.

distribution, this results in the formation of density peaks toward the edge of the distribution, as is seen in both the uniform and Gaussian distributions in Fig. 3. In the nonrelativistic limit, there is no Coulomb shock in planar bunches with cold initial conditions; while in the relativistic limit a strong shock emerges and an initial bunch described by either uniform or a Gaussian density distribution evolves to a two peak structure with one bunch moving to the right and the other to the left (see Fig. 3).

Also apparent in Figs. 3(a) and 3(b) is the fact that stochastic effects are initially strong in simulated density profiles. However, at long times the theoretical density and simulated density agree well. This is a real effect. Specifically, consider the inter-particle distance between the $i$ th and $(i+1)$ th shells denoted as $d_{i}$. For a uniform distribution, order statistics tells us that $d_{i}(0)=\frac{L}{M+1}+\epsilon$ where $L$ is the total width of the distribution, $M$ is the total number of shells, and $\epsilon$ is a stochastic factor roughly of the size $\frac{L}{M+1}$. Thus, due to stochastic fluctuations, we would expect some sheets to be bunched together giving a higher local density than the average and likewise other sheets to be further apart giving a lower local density than the average. This is precisely what is seen with the initial distribution in Fig. 3. However, as these sheets evolve, the relative nonrelativistic acceleration is $\frac{2 q E_{T 1}}{m M}$, so $d_{i}(t)=d_{i}(0)+\frac{q E_{T 1}}{m M} t^{2}$. Given sufficient time, $t \gg \sqrt{\frac{m L}{q E_{T 1}}}$, $d_{i}(t) \approx \frac{q E_{T 1}}{m M} t^{2}$. That is, the interparticle distance (and hence the distribution) is dominated by the space-charge effect and converges to the space-charge predicted distribution everywhere. Of course, if the bunch enters the relativistic regime prior to this smoothing, the stochastic effects will be preserved. We will see such behavior once we add an extraction field, but such behavior requires extremely dense bunches that may not be physically possible in free expansion experiments.

The energy density evolutions in Figs. 3(c) and 3(d) are identical-as expected by Eq. (34). More specifically, while the particles remain nonrelativistic-below the vertical line in Figs. 3(c) and 3(d), the energy density falls for larger kinetic energies as the energy for a planar particle is proportional to $t^{2}$ and therefore neighboring particles move apart in energy-space. However, as particles move into the relativistic regime-above the vertical line in Figs. 3(c) and 3(d), their energy begins to become proportional to $t$. Thus neighboring particles simply scale, and the energy density evolves analogous to nonrelativistic uniform spatial distributions.

\section{Cylindrical symmetry}

Now we consider the expansion of an initially cold charged particle cloud with cylindrical symmetry. In this case, Eq. (13) becomes

$$
v=\frac{\sqrt{2} \zeta y \sqrt{1+\zeta^{2} y^{2}}}{1+2 \zeta^{2} y^{2}} c
$$

where $y^{2}=\ln \left(\frac{r}{r_{0}}\right)=\frac{K}{m c^{2} \rho_{r 02} r_{0}}, \zeta^{2}=\frac{r_{0} \rho_{r 02}}{2}=\frac{r_{0} P_{02}}{2 l_{r 02}}=\frac{r_{0}^{2} \omega_{02}^{2}}{4 c^{2}}$, with $\rho_{r 02}$ coming from Eq. (23). As $l_{r 02} \propto r_{0}$, it should be apparent that $\zeta$ 's dependence on $r_{0}$ is completed determined by $P_{02}\left(r_{0}\right)$.

From Eq. (41) and (14), we find the implicit relation between time and radial position through the integral, 


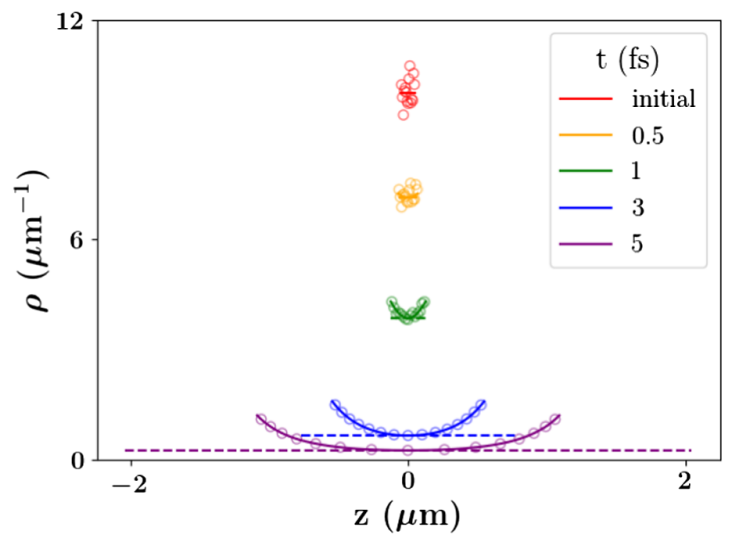

(a)

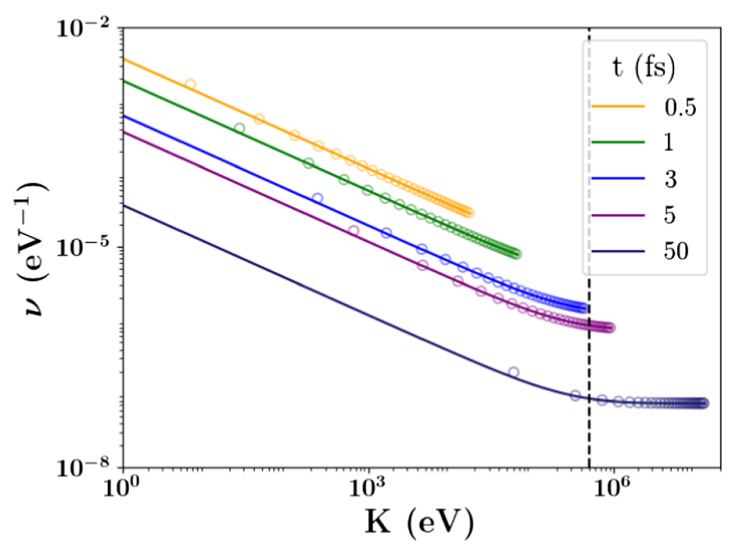

(c)

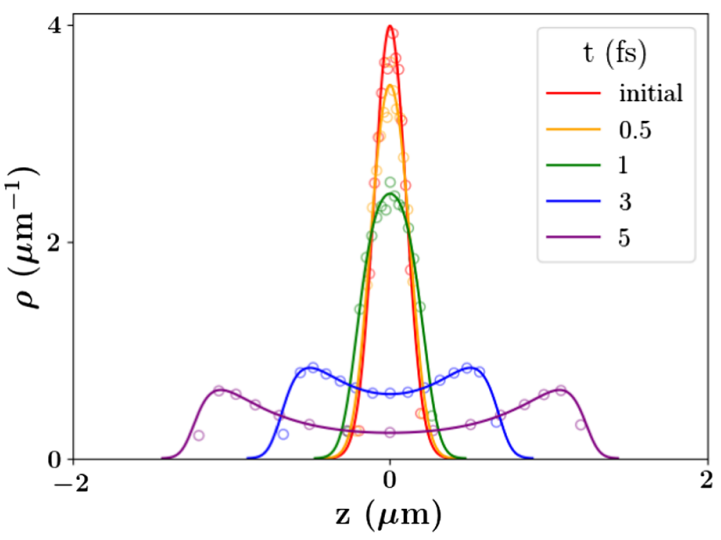

(b)

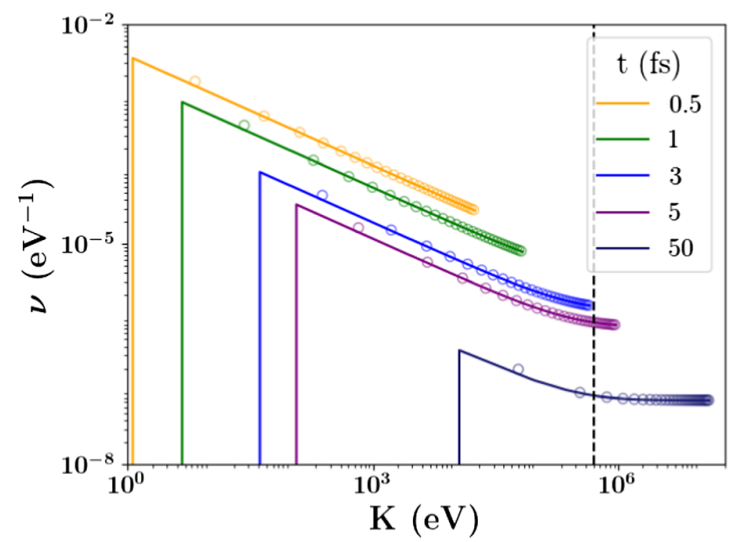

(d)

FIG. 3. Theoretical predictions (solid line) and $M$-shell simulations (hollow dots) of the (a,b) spatial and (c,d) energy densities at (a,b) 4 and (c,d) 5 different times with either (a,c) uniform or (b,d) Gaussian initial spatial distributions. Simulations were accomplished by randomly sampling $M$ macroparticles and advancing them according to the relativistic position equations derived in the text. Notice the log-log scale in the energy plots, and the initial energy densities in (c,d) are not shown as they are delta functions at $K=0$. The simulation had either (a,c) $L=0.1 \mu \mathrm{m}$ or $(\mathrm{b}, \mathrm{d}) \sigma_{L}=0.1 \mu \mathrm{m}, Q_{\mathrm{tot}, 1}=10^{8}$ electrons per $\mu \mathrm{m}^{2}$, and $M=10000$. The extremely high density was chosen as this density does not require significant expansion of the bunch before the onset of relativistic effects. The dotted lines in (a) represents the nonrelativistic prediction of the uniform distribution, and the purple dotted line extends beyond the ends of the $x$-axis but are not shown to keep the scale of expansion closer to what is seen relativistically; notice that the dotted line is tangent to the relativistic distribution at the same time at $z=0$. The deviation of the theory above the dotted line and toward the center of the bunch indicates density freezing due to electrons obtaining luminal speeds whereas the nonrelativistic particles become superluminal. The vertical dashed lines in $(\mathrm{c}, \mathrm{d})$ correspond to the rest energy of an electron. Notice that far below this line, the energy density has one slope, but near this line, the energy density transitions to slope of zero.

$$
t=\frac{2}{\omega_{02}} \int_{0}^{\sqrt{\ln \left(\frac{\tilde{r}}{r_{0}}\right)}} \frac{1+2 \zeta^{2} y^{2}}{\sqrt{1+\zeta^{2} y^{2}}} e^{y^{2}} d y .
$$

To make the connection with previous work, we introduce a generalized Dawson function $\mathcal{F}$ through the definition,

$$
\mathcal{F}(g, x)=e^{-x^{2}} \int_{0}^{x} g(\zeta, z) e^{z^{2}} d z
$$

where $\zeta$ can be written as a function of $x$. Thus the timespatial relation may be expressed as

$$
t=\frac{2}{\omega_{02}} \frac{r}{r_{0}} \mathcal{F}(g(\zeta, y), y)
$$

where

$$
g(\zeta, y)=\frac{1+2 \zeta^{2} y^{2}}{\sqrt{1+\zeta^{2} y^{2}}} .
$$

When $g(\zeta, y)=1$, we reproduce the Dawson function, $F(x)=\mathcal{F}(1, x)$. Specifically when we are in the nonrelativistic regime, we have $2 \zeta y \ll 1$ and $g(\zeta, y) \approx 1$, so Eq. (44) reduces to 


$$
t \approx \frac{2}{\omega_{02}} \frac{r}{r_{0}} F(y)
$$

which is the result we derived previously in the nonrelativistic case. We can write down the derivative of the generalized Dawson function by applying the Leibniz rule

$$
\frac{d \mathcal{F}}{d x}=-2 x \mathcal{F}(g, x)+g(\zeta, x)+\mathcal{F}\left(\frac{\partial g}{\partial \zeta}, x\right) \frac{d \zeta}{d x}
$$

Note that in the nonrelativistic limit, $g(\zeta, y)=1$, and Eq. (47) reduces to the normal Dawson function derivative $\frac{d F}{d x}=-2 x F(x)+1$.

Following the same reasoning as our previous work [32], we can obtain an analytic form for the time dependent density, i.e., the density evolution expression [see Eq. (16)]. Evaluating $r^{\prime}=\frac{d r}{d r_{0}}$ by taking a derivative of Eq. (44) with respect to $r_{0}$, we find,

$r^{\prime}=\frac{r}{r_{0}}\left(1-\frac{2 y \zeta \mathcal{F}_{\partial}}{g(\zeta, y)}\right)\left(1+2 D_{02} y \frac{\mathcal{F}-\zeta \mathcal{F}_{\partial}}{g(\zeta, y)-2 y \zeta \mathcal{F}_{\partial}}\right)$

where $\mathcal{F}$ is shorthand for $\mathcal{F}(g(\zeta, y), y) \mathcal{F}_{\partial}$ is shorthand for $\mathcal{F}\left(\frac{\partial g}{\partial \zeta}, y\right)$, and $D_{02}$ is from Eq. (2). Note $D_{02}$ measures the deviation from a uniform cylindrically symmetric distribution, and for the uniform cylindrically symmetric distribution case it is zero for all values of $r_{0}$ where $\rho_{0}$ is not 0 . Using Eq. (2), the density evolution is found to be,

$\rho_{2}(r ; t)=\frac{r_{0}^{2}}{r^{2}} \frac{\rho_{02}}{\left(1-\frac{2 y \zeta \mathcal{F}_{\partial}}{g(\zeta, y)}\right)\left(1+2 D_{02} y \frac{\mathcal{F}-\zeta \mathcal{F}_{\partial}}{g(\zeta, y)-2 y \zeta \mathcal{F}_{\partial}}\right)}$

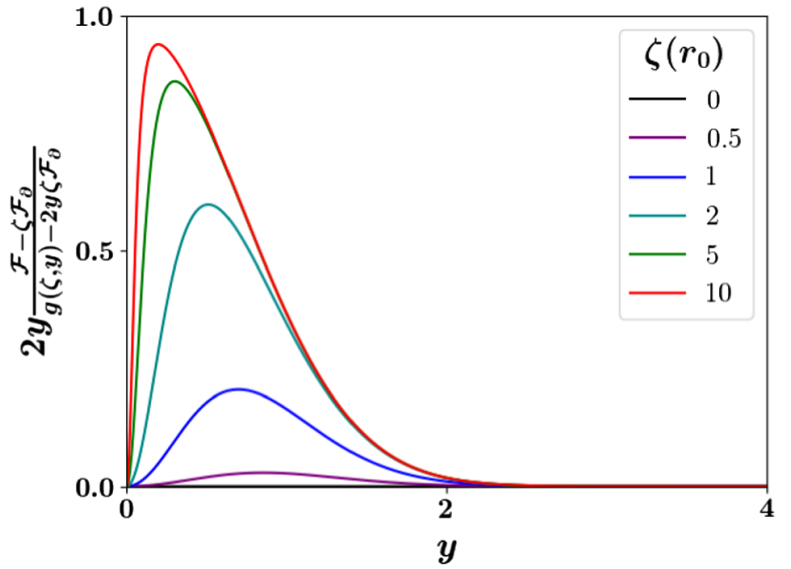

(a) and the energy density evolution is found by substituting Eq. (48) into Eq. (19).

Further note that the crossover time for a specific Lagrangian particle may be obtained by setting Eq. (2) to 0 ; analysis of this equation provides insight into relativistic effects on the onset of the multiflow regime. First, as can be seen in Fig. 4(a), the term $\frac{2 y \zeta \mathcal{F}_{\partial}}{g(\zeta, y)}$, which is zero in the nonrelativistic regime where $\zeta \approx 0$, is always in the range $[0,1)$. This means that the term $\left(1-\frac{2 y \zeta \mathcal{F}_{\partial}}{g(\zeta, y)}\right)$ adds no roots to $r^{\prime}=0$, and all roots must come from $1+2 D_{02} y \frac{\mathcal{F}-\zeta \mathcal{F}_{\partial}}{g(\zeta, y)-2 y \zeta \mathcal{F}_{\partial}}$. Thus, our previous analysis of $D_{02}$ [32] can be extended to the relativistic case.

In Fig. 4(b), we plot the evolution of $2 y \frac{\mathcal{F}-\zeta \mathcal{F}_{\partial}}{g(\zeta, y)-2 y \zeta \mathcal{F}_{\partial}}$; as expected, this analysis reproduces the nonrelativistic function we identified in our previous work when $\zeta=0$. Two observations are apparent from this figure: (1) for a specific $\zeta$, this function has a peak and then asymptotes toward 1 (when $\zeta=0$ ) or 0 and (2) increasing $\zeta$ results in a smaller max for this function. The first observation results in the conclusion that the sign of $2 D_{02} y \frac{\mathcal{F}-\zeta \mathcal{F}_{\partial}}{g(\zeta, y)-2 y \zeta \mathcal{F}_{\partial}}$ is entirely determined by $D_{02}$. As $r^{\prime}>0$ for all $y$ when

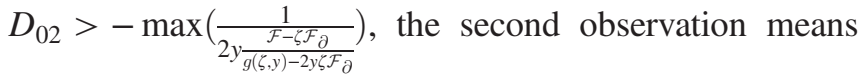
that $D_{02}$ must be more negative than the nonrelativistic case to result in crossover. That is, the relativistic effects make the cylindrical symmetric density evolution more robust against entering into the multi-flow regime.

In Fig. 5, we compare the predictions of Eqs. (49) and (19) to simulations for both uniform and Gaussian initial distributions. We choose the initial radius and radial standard deviation, respectively, to be $1 \mathrm{~cm}$ for $N=1 \times 10^{13}$ electrons $/ \mathrm{cm}$. We again simulate with Warp using the EM solver as well as the 2D version of $M$-shell simulations.

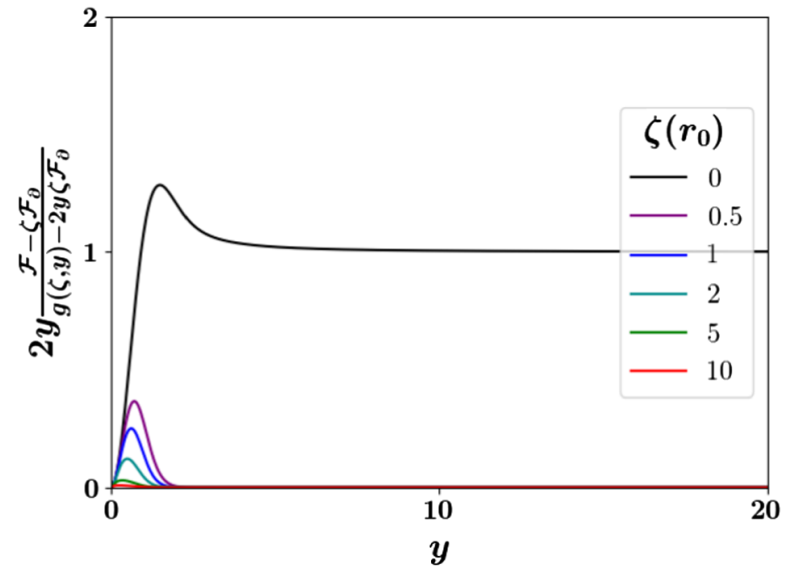

(b)

FIG. 4. Analysis of the functions in $r^{\prime}=\frac{r}{r_{0}}\left(1-\frac{2 y \zeta \mathcal{F}_{\partial}}{g(\zeta, y)}\right)\left(1+2 D_{02} y \frac{\mathcal{F}-\zeta \mathcal{F}_{\partial}}{g(\zeta, y)-2 y \zeta \mathcal{F}_{\partial}}\right)$, where $y=\sqrt{\frac{r}{r_{0}}}$, for various values of $\zeta=\frac{r_{0} \omega_{02}}{2 c}$. Notice that $y$ is monotonic increasing in $r$ for a given $r_{0}$. The term $\frac{2 y \zeta \mathcal{F}_{\partial}}{g(\zeta, y)}$ is plotted in (a) and shows that $\left(1-\frac{2 y \zeta \mathcal{F}_{\partial}}{g(\zeta, y)}\right)$ is greater than 0 for $y>0$. The term $2 y \frac{\mathcal{F}-\zeta \mathcal{F}_{\partial}}{g(\zeta, y)-2 y \zeta \mathcal{F}_{\partial}}$ is plotted in (b). Notice that the max of this second function decreases as $\zeta$ is increased. 


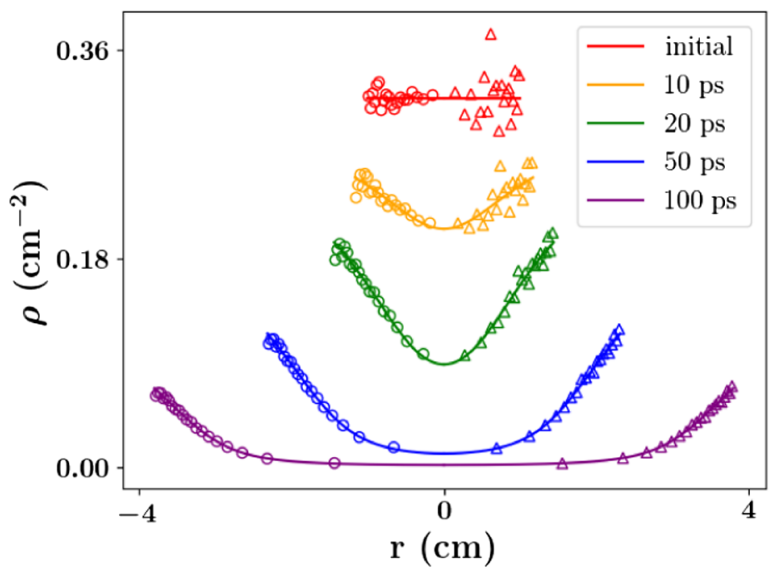

(a)

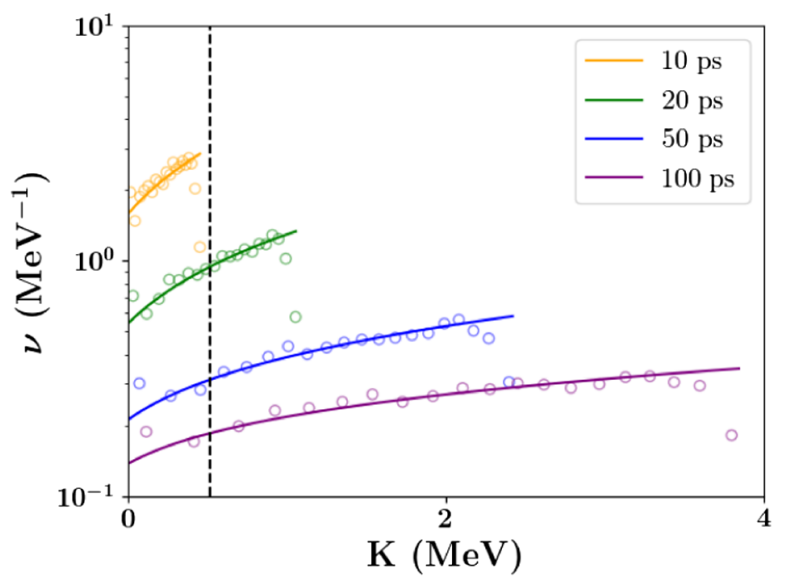

(c)

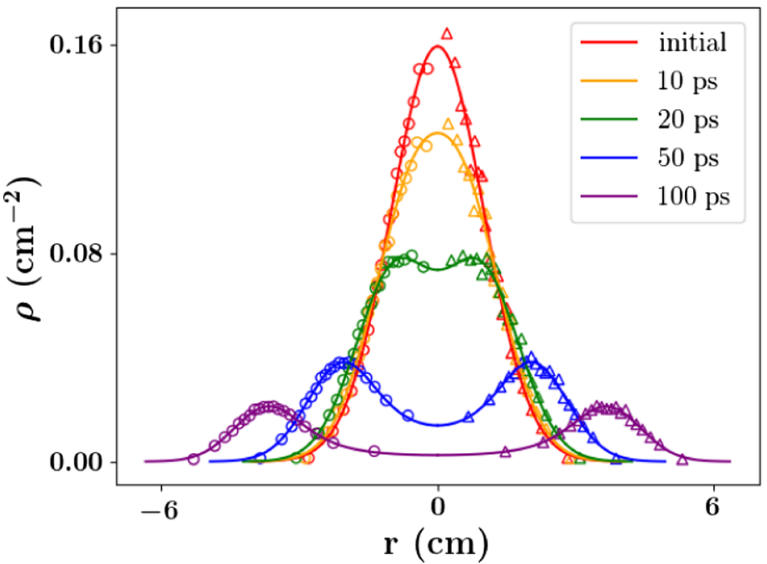

(b)

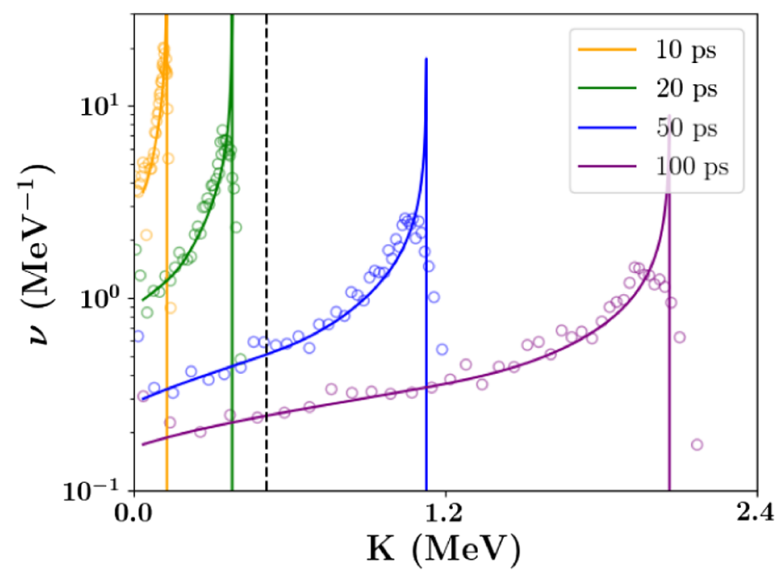

(d)

FIG. 5. Theoretical predictions (solid line), cylindrical $M$-shell simulations (hollow triangles-right), and PIC simulations using an EM solver (hollow circles-left) for (a,b) spatial and (c,d) energy density of (a,c) initially uniform and (b,d) initially Gaussian density at 5 different times for the cylindrically symmetric case. The initial energy distribution is not shown in (c,d) as it is a delta function. PIC simulations were analyzed using the middle $0.2 \mathrm{~m}$ from a simulation of a $2 \mathrm{~m}$-long, 3D distribution of electrons with periodic boundary conditions on the z-axis. The theory spatial density prediction is reflected about the origin as $r$ is strictly greater than 0 and the $\mathrm{m}$-part and PIC simulations are separated into the second and first quadrant, respectively, for purposes of visualization. Parameters were $N=1 \times 10^{13}$ electrons $/ \mathrm{cm}, R_{0}=\sigma_{r, 0}=1 \mathrm{~cm}$, and $M=50000$. Like the planar symmetric case, the density at the center continues to decrease nonrelativistically indicating that the density above the density at the center is due to relativistic effects; however, notice that this peak continues to decrease instead of the evolution freezing at luminal speeds as seen in the planar symmetric case. Further notice that the theoretical energy density is in excellent agreement for the uniform simulation in (c) but only provides an adequate estimate for the initially Gaussian simulation in (d); this is due to crossover, and is discussed further in the text.

For the $M$-shell simulations, the initial radius of the $M$ cylindrical shells are sampled and then evolved according to Eqs. (41) and (46) but with $\omega_{02}$ replaced by $\sqrt{\frac{3 q \Lambda_{s}}{\pi r_{s, 0}^{2} m \epsilon_{0}}}$ where $\Lambda_{s}$ is the charge per unit length contained in the cylindrical shell and $r_{s, 0}$ is the initial radius of the shell. As can be seen in Figs. 5(a) and 5(b), the theory and both simulations agree on the evolution of both the uniform and nonuniform initial distributions. Similar to the planar case, the initial variance about the predicted value can be seen to decrease as the simulations evolve. Again, this indicates that the intershell distances are dominated by the space-charge effects resulting in the later simulations having less statistical variation from the expected distribution. Likewise, the agreement between the theory and simulation energy density evolution is in excellent agreement as can be seen in Fig. 5(c) for the uniform case and Fig. 5(d) for the Gaussian case. Of course, unlike the planar situation where the energy density is independent of the initial spatial distribution, the energy density in the cylindrical case is clearly dependent on the initial spatial distribution.

To validate the density evolution expression, we compare our results to previous results. In the nonrelativistic regime, $2 \zeta y \ll 1 ; \mathcal{F} \rightarrow F$ and $\zeta \mathcal{F}_{\partial} \rightarrow 0$. Thus Eq. (49) reduces to 


$$
\rho_{2}(r ; t)=\frac{r_{0}^{2}}{r^{2}} \frac{\rho_{02}}{1+2 D_{02} y F(y)}
$$

which is the expression we found in our earlier, nonrelativistic work [32].

Similar to the planar symmetric case, we are also interested in the density distribution in specific limits. We were unable to analytically obtain a limit analogous to the limit we found under planar symmetry in Eq. (39) as doing so requires evaluating the value of the modified Dawson function as $\ln \left(\frac{r}{r_{0}}\right)$ goes to infinity. We were able to see the freezing of the dimension in the extremely dense limit where $\zeta \gg 1$ as we have shown in Appendix C. Specifically, the evolution at the edge of the distribution can be approximated by $\rho_{2}(r ; t)=\frac{r_{0}}{r} \rho_{0}$, which is the evolution of the uniform distribution under nonrelativistic conditions in one dimension lower, i.e., 1D. This situation is analogous to the high density $1 \mathrm{D}$ case that causes the edges to essentially immediately become relativistic likewise resulting in evolution of the uniform distribution under nonrelativistic conditions in one-dimension lower, i.e., $0 \mathrm{D}$ or constant. However, this condition, $\zeta \gg 1$, is analogous to the $1 \mathrm{D}$ case when the entire distribution is essentially in the highly relativistic limit. We will shortly show that even in this case, the spherically symmetric evolution can be shown to freeze out a dimension; however, we believe that this freezing happens for cylindrically symmetric distributions regardless of the size of $\zeta$.

\section{Spherical symmetry}

Now we consider the expansion of an initially cold charged particle cloud with spherical symmetry. In this case, Eq. (13) becomes

$$
v=2 \zeta x \frac{\sqrt{g_{1}(x)}}{g_{2}(x)} c
$$

where $x^{2}=1-\frac{r_{0}}{r}=\frac{K}{m c^{2} \rho_{r 03} r_{0}}, g_{1}(x)=1+\zeta^{2} x^{2}, g_{2}(x)=$ $1+2 \zeta^{2} x^{2}, \quad \zeta^{2}=\frac{r_{0} \rho_{r 03}}{2}=\frac{r_{0} P_{03}}{2 l_{r 03}}=\frac{r_{0}^{2} \omega_{03}^{2}}{6 c^{2}}$, and $\rho_{r 03}$ is from Eq. (23). As $l_{r 03} \propto r_{0}^{2}$, it should be apparent that $\zeta \propto r_{0} P_{03}\left(r_{0}\right)$.

From Eq. (41) and (14), we find the implicit relation between time and radial position through

$$
t=\frac{\sqrt{3 / 2}}{g_{1}(1) \omega_{03}}\left(g_{2}(1) \frac{r}{r_{0}} x \sqrt{g_{1}(x)}+T(x)\right)
$$

where $T(x)=\tanh ^{-1}\left(\sqrt{\frac{g_{1}(1)}{g_{1}(x)}} x\right)$. Note that the 1 inside the $g$ functions corresponds to $x$ at infinitely long times, i.e., $\lim _{\frac{r}{r_{0}} \rightarrow \infty} x=1$, so $g_{1}(1)=1+\zeta^{2}$ and $g_{2}(1)=1+2 \zeta^{2}$. This expression is essentially the same expression as derived by Bychenkov and Kovalev, who first derived it for the case of uniform initial density distributions [13]. Our expression differs only in the interpretation of $\omega_{03}$ as ours can be dependent on $r_{0}$ whereas their $\omega_{03}$ is a constant, which is the correct interpretation for the uniform distribution. This difference in interpretation allows us to treat general initial distributions but requires additional consideration when determining the derivative of Eq. (52) with respect to $r_{0}$ as $\omega_{03}^{\prime}=\frac{3 \omega_{03}}{2 r_{0}} D_{03}$, where ' $\equiv \frac{d}{d r_{0}}$, with the $d$ 's in this last expression representing differentiation-not dimensionality of the problem, and $D_{03}$ is from Eq. (2).

We follow the same reasoning as our previous work [32] in order to obtain the density evolution expression. After taking the derivative of Eq. (52) with respect to $r_{0}$, we can solve for $r^{\prime}$ giving

$$
r^{\prime}=\frac{r}{r_{0}} \frac{p_{1}(x)}{g_{1}(1) g_{2}(x)}\left(1+\frac{3}{2} D_{03} \frac{p_{2}(x)}{p_{1}(x)}\right)
$$

where

$$
\begin{aligned}
p_{1}(x)= & g_{1}(1)-\zeta^{2} x^{2} \frac{g_{1}(x)}{g_{1}(1)}+2 \frac{\zeta^{4} x^{2}}{g_{1}(1)} \frac{r_{0}}{r}\left(1+\frac{1}{g_{1}(1)}\right) \\
& +\frac{r_{0}}{r} 3 \zeta^{2} x \sqrt{\frac{g_{1}(x)}{g_{1}^{3}(1)}} T(x)
\end{aligned}
$$

and

$$
\begin{aligned}
p_{2}(x)= & \frac{g_{1}(x)+2 \zeta^{4} \frac{r_{0}}{r}}{g_{1}(1)} x^{2} \\
& +\frac{r_{0}}{r}\left(1+4 \zeta^{2}\right) x \sqrt{\frac{g_{1}(x)}{g_{1}^{3}(1)}} T(x) .
\end{aligned}
$$

Plugging Eq. (53) into Eq. (16) we obtain the evolution of the density distribution

$$
\rho_{3}(r ; t)=\frac{r_{0}^{3}}{r^{3}} \frac{g_{1}(1) g_{2}(x) \rho_{0}}{p_{1}(x)+\frac{3}{2} D_{03} p_{2}(x)}
$$

and the evolution of the energy density distribution can be obtained by plugging Eq. (56) into Eq. (19).

As in the cylindrical symmetric case, we analyze Eq. (53) for crossover events, i.e., $r^{\prime}=0$. As $g_{1}(x)>1$ and $g_{2}(x)>1$ for all $x$, we plot $p_{1}(x)$ in Fig. 6(a) demonstrating that $p_{1}(x)$ does not provide roots for $r^{\prime}$. Like our previous work and the cylindrical case, this reduces to an analysis of the function, in this case $\frac{p_{2}(x)}{p_{1}(x)}$, times the deviation from uniformity measure, $D_{03}$. As can be seen in Fig. 6(b), this function again has the same two properties as the cylindrical function: namely (1) it is strictly positive approaching 1 at long times and (2) increasing $\zeta$ reduces the value of the function for a given $x$. Interestingly, around $\zeta=0.7$, this function becomes 


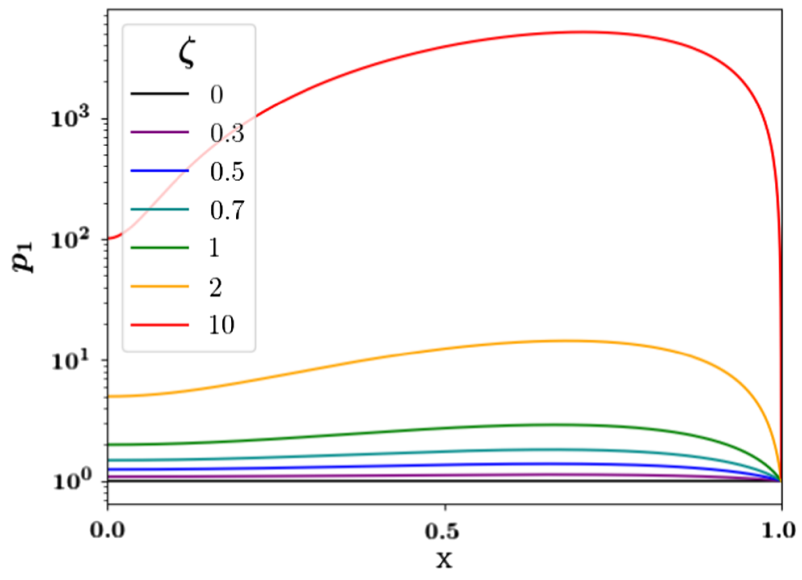

(a)

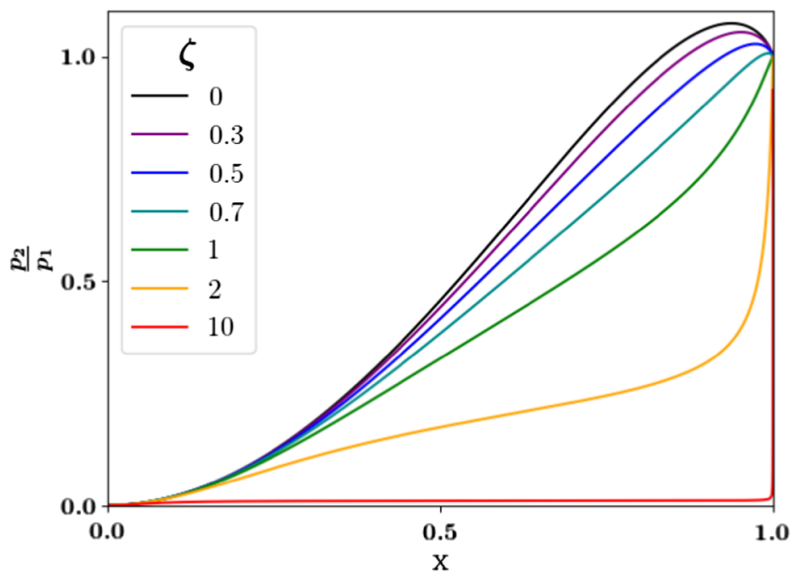

(b)

FIG. 6. Analysis of the functions in $r^{\prime}=\frac{r}{r_{0}} \frac{p_{1}(x)}{g_{1}(1) g_{2}(x)}\left(1+\frac{3}{2} D_{03} \frac{p_{2}(x)}{p_{1}(x)}\right)$, where $p_{1}, p_{2}, g_{1}$, and $g_{2}$ are defined in the text. Notice that $x^{2}=1-\frac{r_{0}}{r}$ has a range $[0,1]$ with is $x=0$ occurring at $t=0$ and $x=1$ occurring at $t=\infty$. The term $p_{1}(x)$ is plotted in (a) and shows that $p_{1}(x)$ is greater than 0 for $x>0$. The term $\frac{p_{2}}{p_{1}}$ is plotted in (b). Notice that the value of this second function for a specific $x$ in $(0,1)$ decreases as $\zeta$ is increased.

monotonically increasing and the maximum becomes 1 at long times. So again, relativistic effects make the density evolution more robust to multiflow onset.

In Fig. 7, we compare the predictions of Eqs. (56) and (19) to simulations for both uniform and Gaussian initial distributions of $N=1 \times 10^{13}$ electrons. The simulations have $R=1 \mathrm{~cm}$ (uniform) or $\sigma_{r}=1 \mathrm{~cm}$ (Gaussian). We again simulate with Warp using the EM solver and as well as the 3D version of $M$-shell simulations. For the $M$-shell simulations, the initial radius of the $M$ spherical shells are sampled and then evolved according to Eqs. (51) and (52) but with $\omega_{03}$ replaced by $\sqrt{\frac{3 q Q_{s}}{4 \pi r_{s, 0}^{3} m \varepsilon_{0}}}$ where $Q_{s}$ is the charge contained in the shell and $r_{s, 0}$ is the initial sampled radius of the shell. As can be seen in Figs. 7(a) and 7(b), the theory captures the evolution of both the uniform and nonuniform initial distributions. Similar to both the planar and cylindrical cases, the initial variance around the theoretical value primarily seen in the uniform distribution decreases as the distribution expands. Also like the other two cases examined, the energy distributions from theory seen in Figs. 7(c) and 7(d) are in excellent agreement with simulation; furthermore, the initial spatial distribution is again seen to influence the energy density evolution as it did under cylindrical symmetry.

For further validation, we compare Eq. (56) to the expression derived by Bychenkov and Kovalev. Their expression detailed the relativistic density evolution for the uniform distribution [13], $\rho_{\text {unif }}(r ; t)$, which should be equivalent to our expression when $D_{03}=0$. In this case, Eq. (56) reduces to

$$
\rho_{3 \text { unif }}(r ; t)=\frac{r_{0}^{3}}{r^{3}} \frac{g_{1}(1) g_{2}(x) \rho_{0}}{p_{1}(x)} .
$$

This expression for the density evolution for uniform initial conditions is identical to the expression published in the English translation of Bychenkov and Kovalev except for an obvious typo in that work [13].

Next, we compare this expression to our previous, nonrelativistic expression. In the nonrelativistic regime $2 \zeta^{2} \ll 1$. Unlike the planar and cylindrical cases, the spherical model need never enter the relativistic regime and therefore this model may be relevant for all time. In this nonrelativistic regime, Eq. (56) reduces to

$$
\rho_{3 N R}(r ; t)=\frac{r_{0}^{3}}{r^{3}} \frac{\rho_{0}}{1+\frac{3}{2} D_{03}\left(x^{2}+\frac{r_{0}}{r} x \tanh ^{-1} x\right)}
$$

which is identical to the nonrelativistic expression we previously derived but with $D_{03}=\frac{2}{3} D_{3}$ in our previous notation [32].

Again we would like to analyze specific limits of the density evolution; fortunately, under spherical symmetry we can analyze the long time limit. In Appendix B we show that

$$
\lim _{\frac{r}{r_{0} \rightarrow \infty}} \rho_{3}=\frac{r_{0}^{3}}{r^{3}} \rho_{x 3}\left(r_{0}\right)
$$

where $\rho_{x 3}\left(r_{0}\right)=\frac{1+3 \zeta^{2}+2 \zeta^{4}}{1+\frac{3}{2} D_{03}} \rho_{03}$ is entirely determined by the initial conditions. Notice, the prefactor in $\frac{1+3 \zeta^{2}+2 \zeta^{4}}{1+\frac{3}{2} D_{03}}$ is essentially 1 in the center where $\zeta \approx 0$ and $D_{03} \approx 0$, but that this value increases as $r_{0}$ increases. The time evolution of $\rho_{x 3}$ and the predicted asymptote for this quantity can be seen in Fig. 8. For the uniform distribution, the increase in $\rho_{x 3}$ as a function of $r_{0}$ is quartic as $D_{03}=0$ for all values 


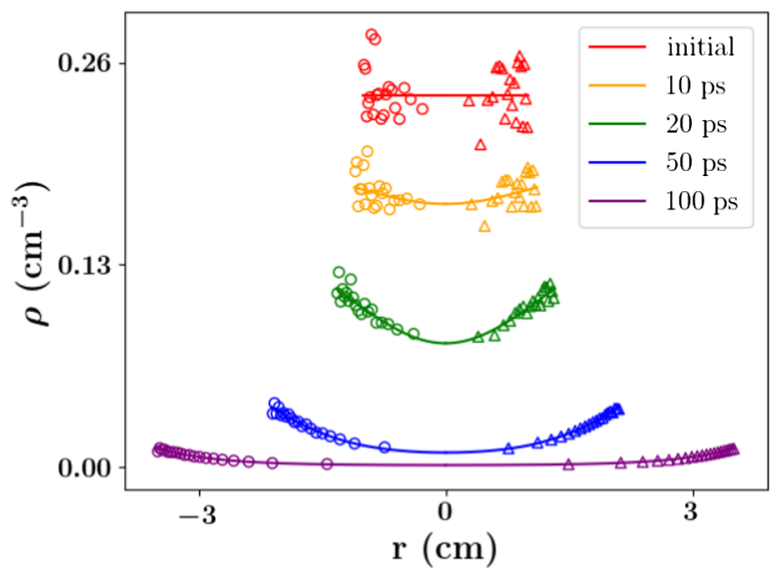

(a)

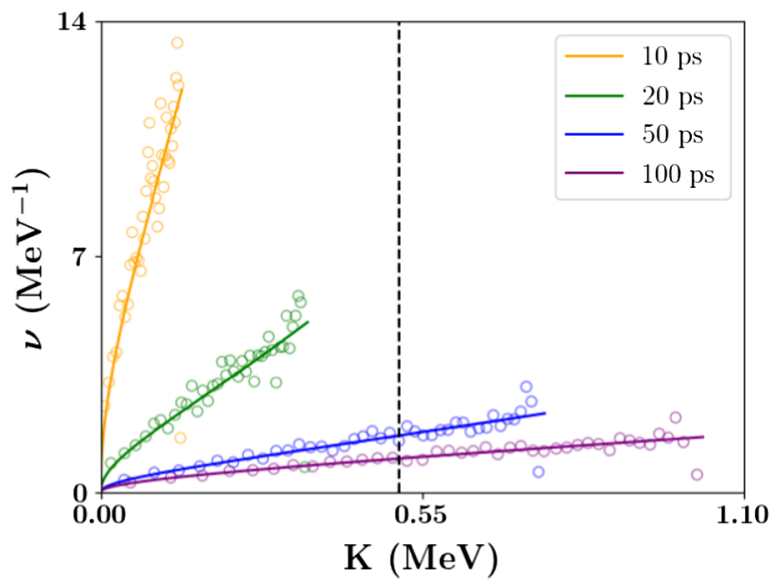

(c)

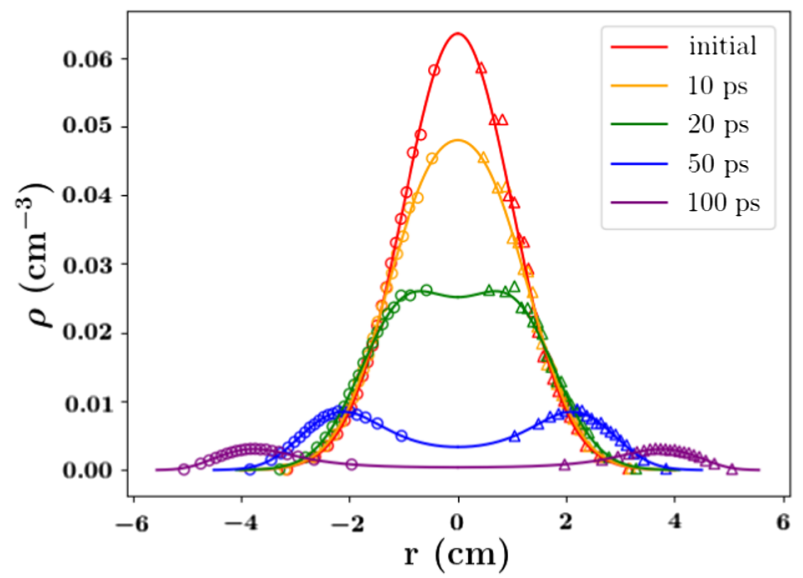

(b)

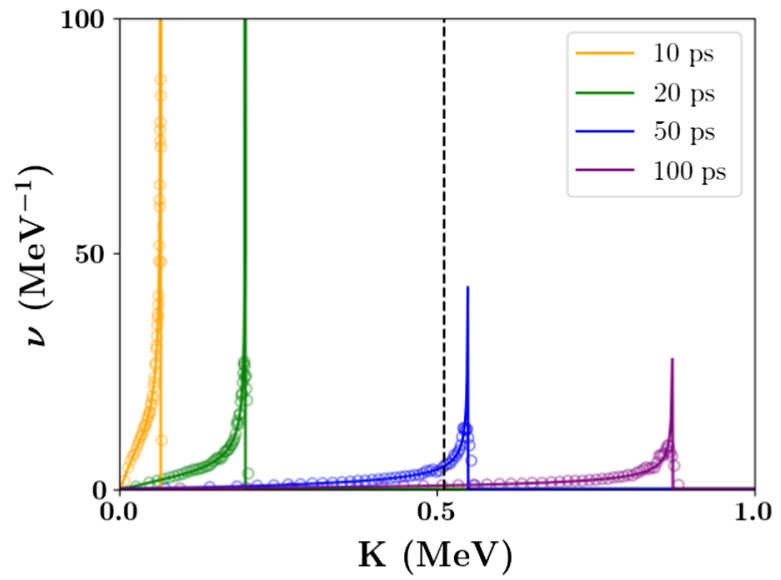

(d)

FIG. 7. Theoretical predictions (solid line), spherical $M$-shell simulations (hollow triangles-right), and PIC simulations using an EM solver (hollow circles-left) of (a,c) initially uniform and (b,d) initially Gaussian (a,b) spatial and (c,d) energy density at 5 different times for the spherically symmetric case. The spatial theory is reflected about the origin as $r$ is strictly greater than 0 and the $M$-shell and PIC simulations are separated into the first and second quadrant, respectively, for purposes of visualization. Parameters were $N=1 \times 10^{13}$ or $N=3 \times 10^{13}$ electrons for the uniform and Gaussian case, respectively, $R_{0}=\sigma_{r, 0}=1 \mathrm{~cm}$, and $M=50000$. Like the planar and cylindrical symmetric uniform cases, the density at the center in (a) continues to decrease nonrelativistically indicating that the density above the density at the center is due to relativistic effects; similar to the cylindrical symmetric case, the relativistic density continues to evolve. However, the evolution of the peak decreases much faster than the cylindrical case-which is discussed in detail in the text. The peak in the Gaussian density in (b) is a combination of both relativistic and previously described bunching effects. Likewise, the evolution of the energy density is captured by theory as seen in (c) and (d) - arguably better than the energy density evolution seen in the cylindrical case.

of $r_{0}$. In real distributions, though, there should be a value for $r_{0}$ where $D_{03}=-\frac{2}{3}$, and we see that $\rho_{x 3}$ has a zero in the denominator. This violates the assumptions made in the derivation of $\rho_{x 3}$, and inspection of Appendix B shows that $r^{\prime}$ becomes 0 in the locality of $D_{03}=-\frac{2}{3}$ suggesting a violation of the laminar fluid assumption. For the Gaussian distribution, roughly $80 \%$ of the distribution is contained within the radius where $D_{03}\left(r_{0}\right)=-\frac{2}{3}$ suggesting that at least the majority of the distribution is captured by this theory. Furthermore, the precise shape for $\rho_{x 3}\left(r_{0}\right)$ for a uniform and Gaussian distribution may be seen in Fig. 9; however, in the $1 \mathrm{D}$ case, $\rho_{01}$ truly asymptotes whereas here $\rho_{03}$ continues to decrease eventually with the uniformlike behavior of $\frac{r_{0}^{3}}{r^{3}}$. This difference is largely due to the fact that all particles asymptote to the same velocity, $c$, in the planar case but different velocities in the spherical case.

This analysis leads to the second $\operatorname{limit}$ of $\lim _{\zeta \rightarrow \infty}$, an unphysical limit, analogous to the 1D and cylindrically symmetric cases where we saw the density at the edge lose dimensionality. Likewise, in the spherical-symmetric case we show in Appendix $\mathrm{C}$ that

$$
\lim _{\zeta \rightarrow \infty} \rho_{3}(r ; t)=\frac{r_{0}^{2}}{r^{2}} \rho_{03}
$$




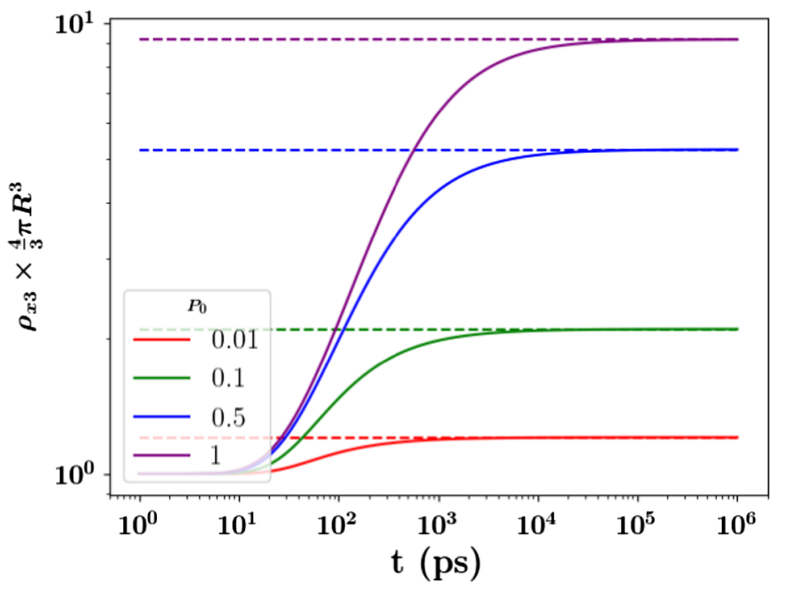

(a)

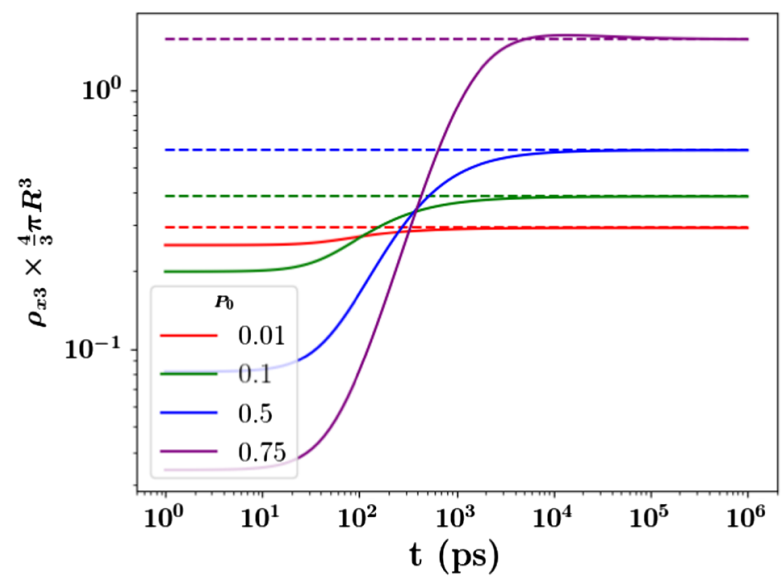

(b)

FIG. 8. The time evolution (solid lines) of $\rho_{x 3}$, defined in the text, for a distribution with $1.602 m C$ in (a) an initially uniform sphere of width $1 \mathrm{~mm}$ and (b) an initially Gaussian sphere with standard deviation of $1 \mathrm{~mm}$. The dashed lines indicate the corresponding highlyrelativistic limit of $\rho_{x 3}$ obtained analytically. Again, the formation of the density peak from these relativistic considerations is apparent in the graphs. Similar to how the planar symmetric density freezes, $\rho_{x 3}$ can be seen to asymptote; however, this is due to the Lagrangian particle reaching their terminal velocity, the difference of which is relativistically contracted, as described in the text.

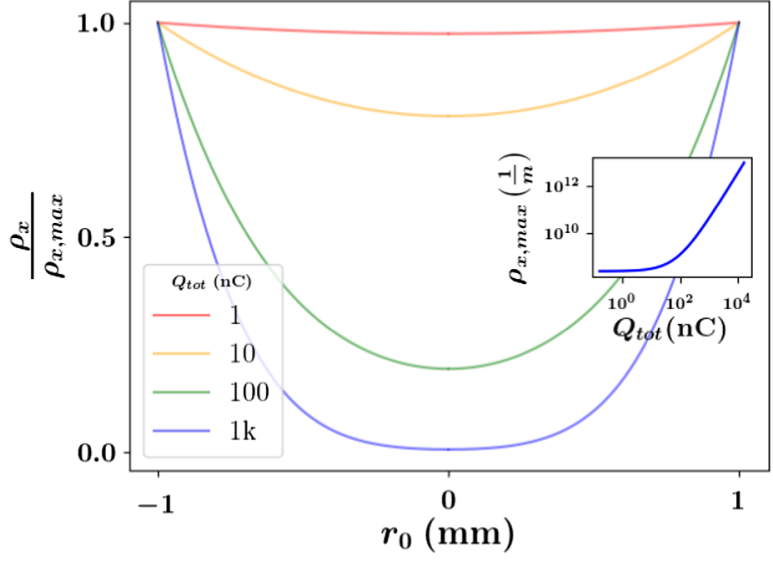

(a)

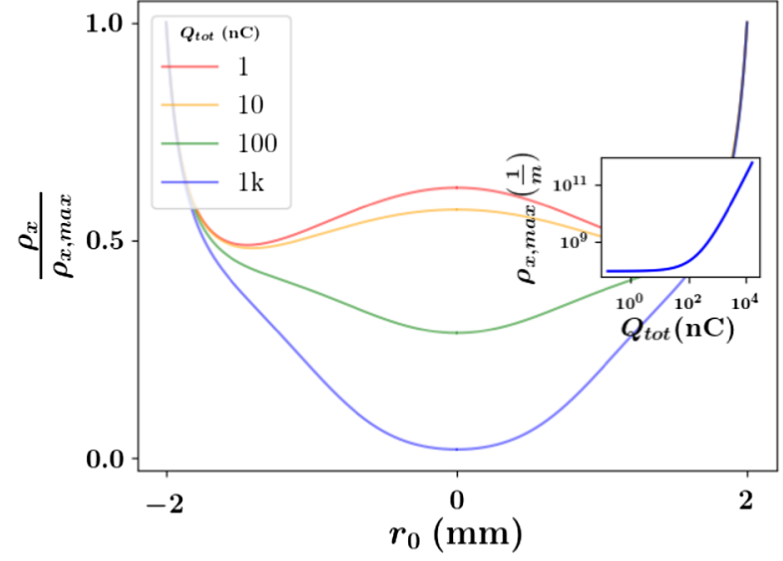

(b)

FIG. 9. The shape of $\frac{\rho_{x 3}}{\rho_{x 3, \max }}$ for various values of $Q_{\mathrm{tot}, 3}$ in (a) an initially uniform distribution with radius of $1 \mathrm{~mm}$ and (b) an initially Gaussian distribution with standard deviation of $1 \mathrm{~mm}$ as a function of the initial radial coordinate. This, as well as the inset figure describing the growth of the maximum value of $\rho_{x 3}$, show the onset of the relativistic regime corresponding to roughly $24 \frac{C}{m}$. The main graph also shows that the peaks sharpen as the density increases. For the Gaussian case, this distribution diverges near $r_{0}=2$. This divergence is an indication that the laminar fluid assumption is being violated, and therefore the nonuniform asymptote for $\rho_{x 3}$ should be taken as a gross approximation; nonetheless, the expression derived in the text do capture the shape of freely-expanding relativistic Gaussian distribution in the simulation presented in Fig. 7.

which is again the uniform density evolution of a symmetric distribution in one dimension less than the one being considered. While appearing unphysical, this does have some physical significance. This suggests that such distributions evolve toward $\rho_{x}\left(r_{0}\right)$ in such a way that the factor in the denominator exactly cancels out the factor of $\frac{r_{0}}{r}$, i.e., $r^{\prime} \approx 1$ early on. However, as time progresses, the evolution shifts toward the decay of the uniform distribution in the appropriate dimension.

\section{EXTRACTION FIELD IN THE PLANAR MODEL}

It is straightforward to introduce an extraction field in the planar model, and this is relevant to dynamics of electron density distributions in the pancake bunches used in ultrafast electron microscopy. The equations for this case are nearly identical to the equations derived for the planar model in the absence of an extraction field with the single replacement 


$$
P_{01} \rightarrow P_{01}+\frac{E_{a}}{E_{T 1}}
$$

which may also be written as

$$
\bar{\rho}_{01} \rightarrow \bar{\rho}_{01}+\frac{E_{a}}{z_{0} E_{T 1}}
$$

- $\mathrm{a}$ form that is especially helpful in the energy density expression, Eq. (19). Equivalently, this may be written as

$$
\rho_{r 01} \rightarrow \rho_{r 01}+\rho_{a}
$$

where $\rho_{r 01}$ is from Eq. (23) and $\rho_{a}=\frac{e E_{a}}{m c^{2}}$, which also can be interpreted as a new length scale, $l_{a}=\frac{m c^{2}}{e E_{a}}$, associated with the extraction field. The one caveat that is necessary to note is that symmetry is broken by the applied field; thus the analysis of the energy distribution requires the full treatment of $\mathcal{R}\left(K_{1} ; t\right)$ so that Eq. (18) becomes

$$
\nu_{1}(K ; t)=\sum_{z_{0} \in \mathcal{R}} \frac{1}{4 m c^{2}} \frac{l_{r 01}}{c t} \frac{1+\frac{K_{1}}{m c^{2}}}{\sqrt{\left(1+\frac{K_{1}}{m c^{2}}\right)^{2}-1}} .
$$

In some regimes, $\mathcal{R}$ will have 2 values, while in others, there will only be one. In the broken symmetry cases, this results in the density discretely jumping to half of its value where the energy of the particles accelerating in the opposite direction from the applied field is maximal.

We choose the applied field to be in the positive $z$ direction. For applied fields, $E_{a}$, with $E_{a}>E_{T 1}\left(l_{a}<l_{r 01}\right)$ the applied field is sufficiently strong to overcome the space charge field throughout the bunch, hence accelerating all of the particles in the same direction. For smaller applied fields, $E_{a}<E_{T 1}\left(l_{a}>l_{r 01}\right)$, particles in the negative $z$ regions of the initial charge distribution may experience a stronger intrinsic space charge field than can be overcome

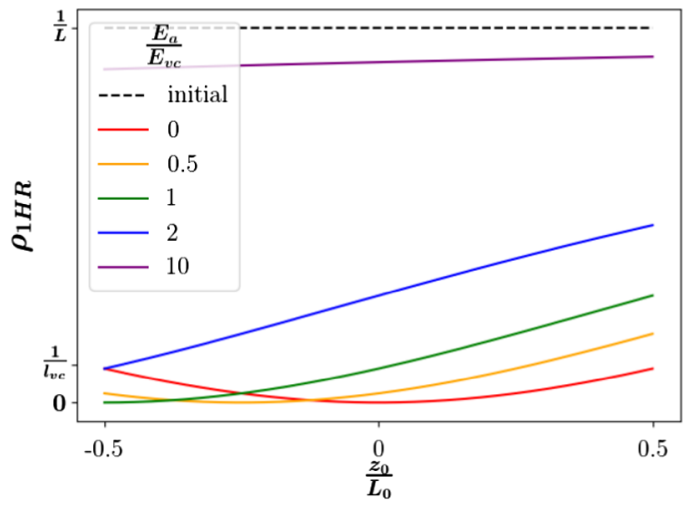

(a) uniform by the applied field. In this case the initial distribution breaks up into two bunches moving in opposite directions. This is the virtual cathode limit defined by Valfells et al. [36]. We also point out that $l_{r 01}$ corresponds to the length scale of this limit. The fraction of charge in the bunch that moves in the positive and negative $z$ direction is simply $\frac{1}{2}\left(1 \pm \frac{E_{a}}{E_{T 1}}\right)$, respectively. The form of the two bunches is given by Eq. (31), with the substitution given in Eq. (61). Taking the relativistic limit of this expression, we obtain the asymptotic form of the two bunches

$\rho_{1 H R}(z ; t) \rightarrow \frac{\rho_{01}}{\frac{1}{l_{r 01}}\left(P_{01}+\frac{E_{a}}{E_{T 1}}\right)^{2}+\rho_{01}}\left(P_{01}+\frac{E_{a}}{E_{T 1}}\right)^{2}$

and the asymptotic form for the uniform and Gaussian distributions for various applied fields are demonstrated in Fig. 10. Equation (65) can be written in terms of the plasma period, $\omega_{01}$, and other terms, but we find that both the applied field scale, $E_{T 1}$, and the associated length scale, $l_{r 01}$, are more apparent in this formulation.

For the case of a uniform initial distribution $\rho_{01}=\frac{1}{2 L}$ on the domain $[-L, L]$, the field of a particle at position $z_{0}$ is given by

$$
E_{01}\left(z_{0}\right)+E_{a}=E_{T 1} \frac{z_{0}}{L}+E_{a} .
$$

Setting the total field to zero gives the point at which the pulse breaks up into two pulses,

$$
z_{x}=-\frac{E_{a}}{E_{T 1}} L
$$

Notice that as $E_{a}$ goes above $E_{T 1}, z_{x}$ goes below $-L$ indicating that there is no split in the pulse consistent with prior analysis. As long as $E_{a}<E_{T 1}$, the peak density of

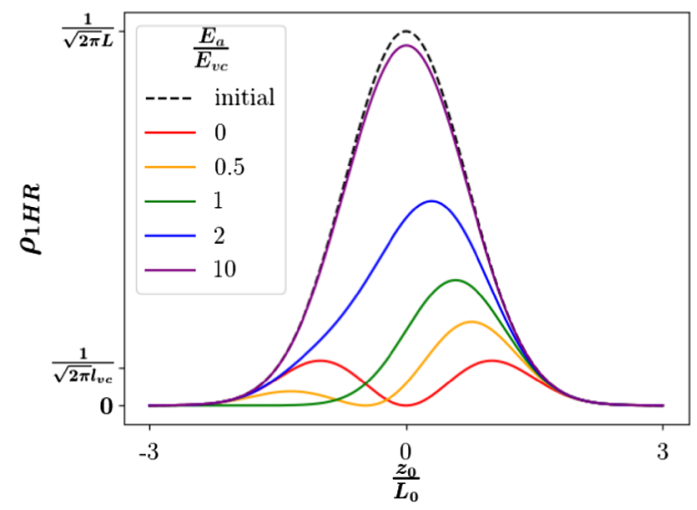

(b) Gaussian

FIG. 10. Theoretical planar symmetric asymptotic distributions (solid lines) plotted against the initial position for various applied fields, measured in multiples of $E_{T 1}=\frac{Q_{\text {tot, }}}{2 \epsilon}$. The black dotted line indicates the initial distribution. Notice that in both cases, the actual distribution is located at $z(t)$ at time $t$, but as this has a one-to-one relation with $z_{0}$, we use the initial location for the sake of comparison. 
each pulse after it has gone relativistic can be calculated from Eq. (65) and is

$$
\rho_{\text {1right } / \text { left }}=\frac{1}{2 L+l_{r 01}\left(\frac{E_{a}}{E_{T 1}} \pm 1\right)^{-2}}
$$

again with the positive, rightward pulse corresponding to the + and the negative, leftward pulse corresponding to the - .

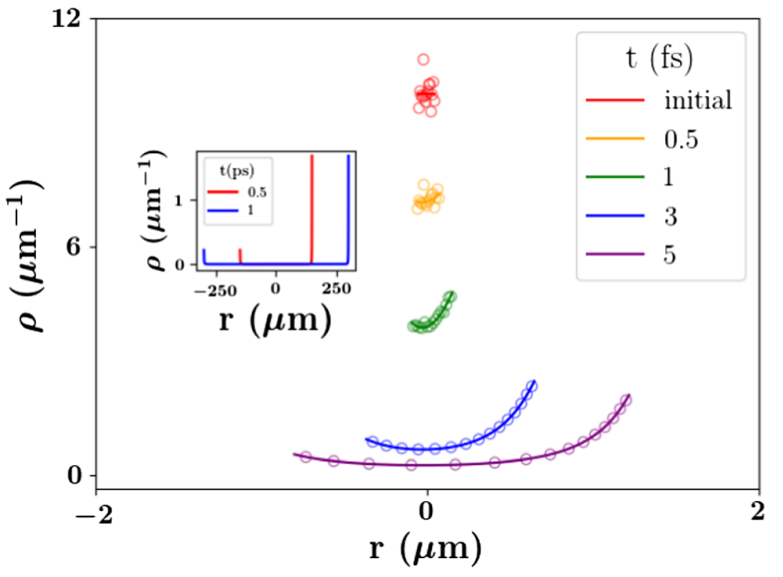

(a) $E_{a}=0.5 E_{T 1}$

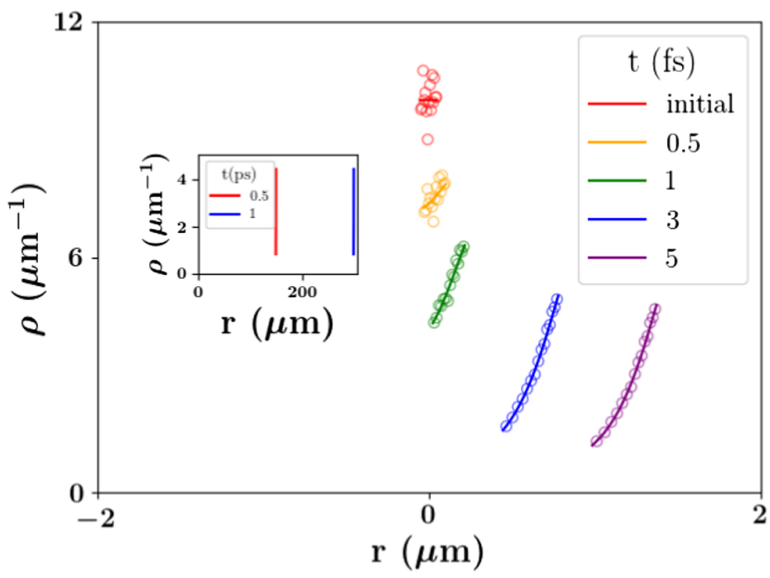

(c) $E_{a}=2 E_{T 1}$
The effect of the extraction field on the time-dependent density evolution can be seen in Figs. 11, 12, and 13 which show the evolution of initially uniform and Gaussian spatial distributions and both of their energy density evolutions, respectively, in the presence of various extraction fields. First, notice that the inclusion of a nonzero $E_{a}$ breaks the symmetry of the left and right pulses and that we can see that the double pulses are replaced by a single pulse as the applied field crosses the virtual cathode limit, $E_{a}=E_{T 1}$. As $E_{a}$ is increased beyond $E_{T 1}$, all Lagrangian particles

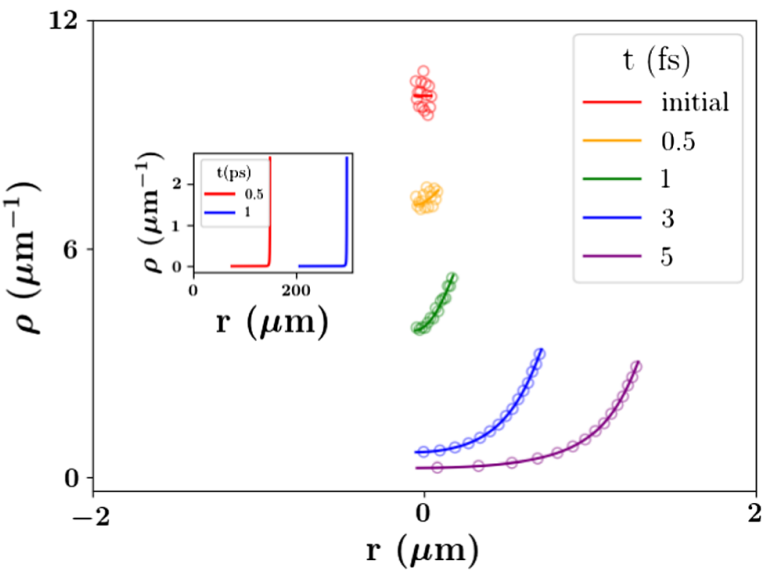

(b) $E_{a}=E_{T 1}$

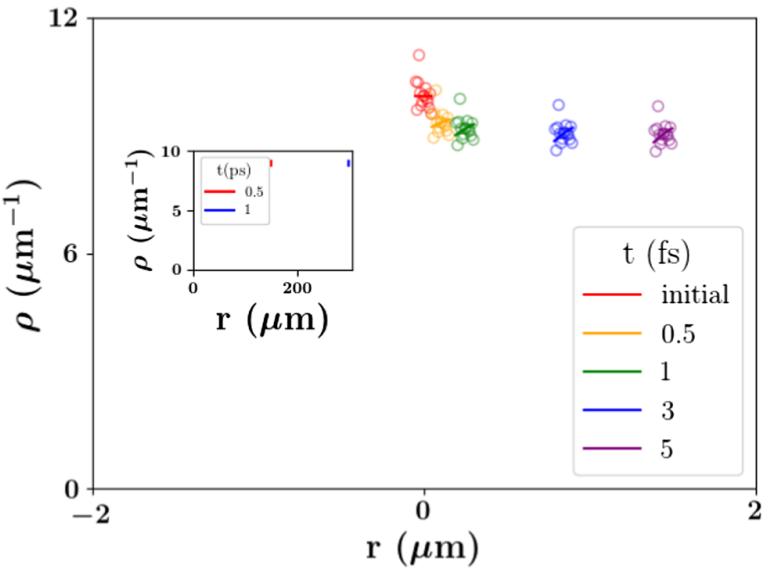

(d) $E_{a}=10 E_{T 1}$

FIG. 11. Theoretical predictions (solid line) and $M$-shell simulations (hollow dots) of the planar symmetric density at 4 different times with different applied extraction fields: (a) $E_{a}=0.5 E_{T 1}$, (b) $E_{a}=E_{T 1}$, (c) $E_{a}=2 E_{T 1}$, and $E_{a}=10 E_{T 1}$ where $E_{T 1}=\frac{Q_{\text {tot. }}}{2 \epsilon_{0}}$ and $Q_{\mathrm{tot}, 1}$ is the total surface-charge density. The simulation had $L=0.1 \mu \mathrm{m}$ and $Q_{\mathrm{tot}, 1}=1 \times 10^{20}$ electrons per $\mathrm{m}^{2}$ and $M=10000$. The extremely high density was chosen as this density not require significant expansion of the bunch before the onset of relativistic effect. Simulations were similar to those described in Fig. 3, and again the inset graphs show the theoretic density at 2 much later times $(0.5$ and $1 \mathrm{ps})$. While the distributions at later times appear deltalike, they do have at least the same width as seen at earlier time-however, this width is much smaller than the scale resulting in the deltalike behavior at later times. Notice that in the main plot the scales are consistent among graphs. Also notice that the extraction fields have little effect on where the front of the bunches are after 1 ps but have dramatic effect on the bunch distribution - this is partially an artifact of the high density of the initial distribution that results in the front of the distribution relatively quickly becoming relativistic with or without an extraction field. In addition to the shape of the asymptotic density, the extraction field determines to what extent the initial variance about the meanfield theory prediction is lost (an effect explained in the text.) 
eventually become relativistic, and the density "lifts" away from the axis. Eventually (not shown), the extraction field should be strong enough that no appreciable expansion occurs and the initial distribution is simply displaced at the speed of light; this can be shown to occur when $E_{a} \gg E_{T 1}$.

Also as can be seen in Figs. (11) and (12), the initial stochastic variation in the density is lost for simulations of sufficiently low extraction field but is retained for $E_{a} \geq 10 E_{T 1}$. This is due to the same effect discussed in the planar model without an electric field; however, the relativistic timescale needs to be adjusted, namely

$$
\tau_{\text {rel }}=\frac{l_{r 01}}{c}\left|P_{01}+\frac{E_{a}}{E_{T 1}}\right|^{-1}
$$

When $\tau_{\text {rel }} \gg \tau_{\text {exp }}$, we again have the case where the expansion dynamics dominate and the inter-particle spacings essentially are equivalent to the interparticle spacings determined by theory. However, once $\tau_{\text {rel }} \ll$ $\tau_{\text {exp }}$, the inter-particle spaces do not expand sufficiently to overcome the initial stochastics and the variance is preserved. The new wrinkle is that $\tau_{\text {rel }}$ can be reduced by simply increasing the extraction field. Therefore, we do in fact see a distribution evolve that retains the initial variance, i.e., $E_{a}=10 E_{T 1}$ in Fig. 11 , as for that simulation $\tau_{\text {rel }} \ll \tau_{\text {exp }}$.

Moreover, the influence of the extraction field is important in the highly relativistic regime not only for influencing the timescale but also influencing the asymptotic distribution. Specifically, the effect of the extraction field in the 1D model is apparently not to accelerate the front of the distribution, i.e., all simulations had the front of the bunch traveling near the speed of light, but instead to shape the eventual distribution as can be seen in Figs. 11 and (12).

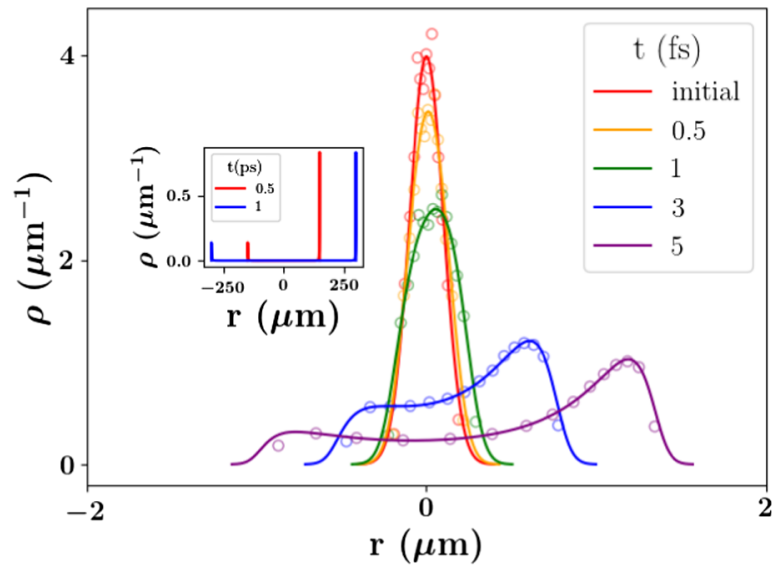

(a) $E_{a}=0.5 E_{T 1}$

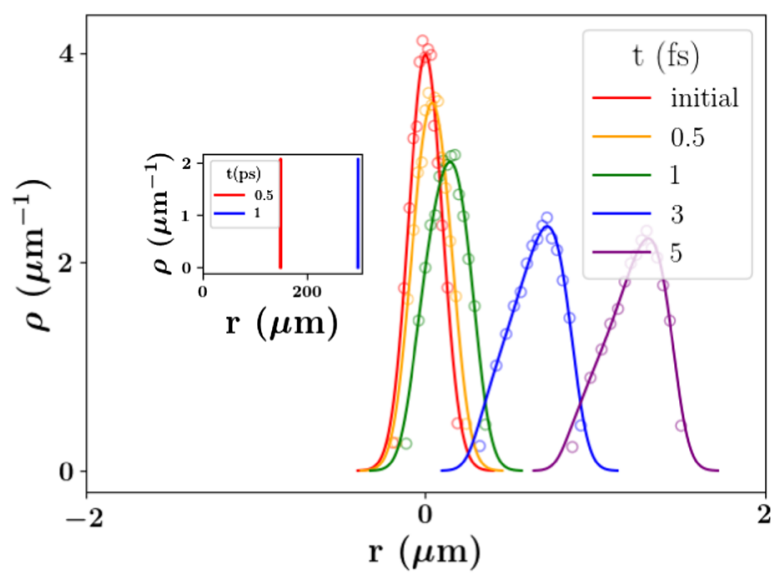

(c) $E_{a}=2 E_{T 1}$

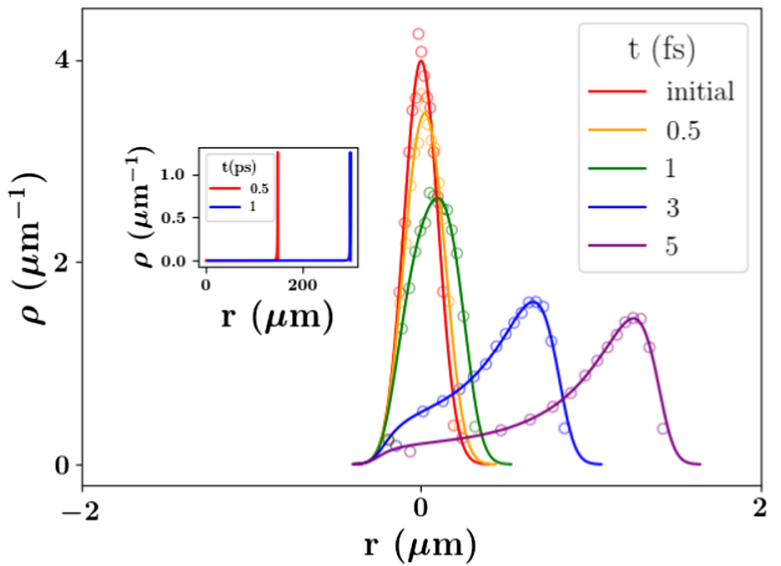

(b) $E_{a}=E_{T 1}$

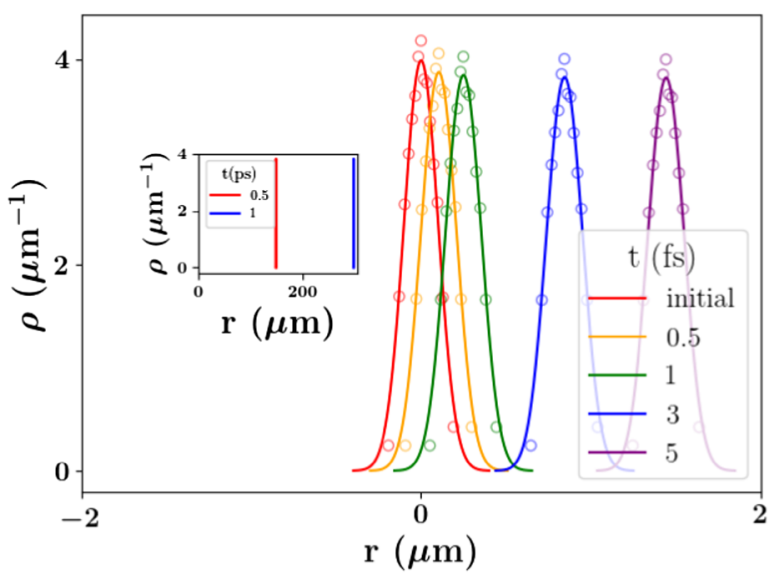

(d) $E_{a}=10 E_{T 1}$

FIG. 12. Theoretical predictions (solid line) and $M$-shell simulations (hollow dots) of the planar symmetric density at 4 different times with different applied extraction fields: (a) $E_{a}=0.5 E_{T 1}$, (b) $E_{a}=E_{T 1}$, (c) $E_{a}=2 E_{T 1}$, and $E_{a}=10 E_{T 1}$ where $E_{T 1}=\frac{Q_{\text {tot.1 }}}{2 \epsilon_{0}}$ and $Q_{\mathrm{tot}, 1}$ is the total surface-charge density. Parameters and simulations are analogous to those described in Fig. 11 excepting the initial Gaussian distribution with $\sigma_{r}=0.1 \mu \mathrm{m}$. 


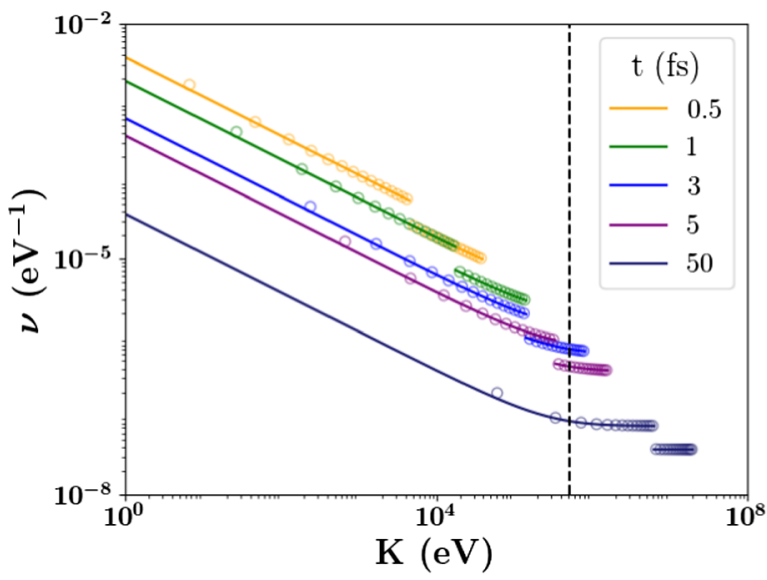

(a) $E_{a}=0.5 E_{T 1}$

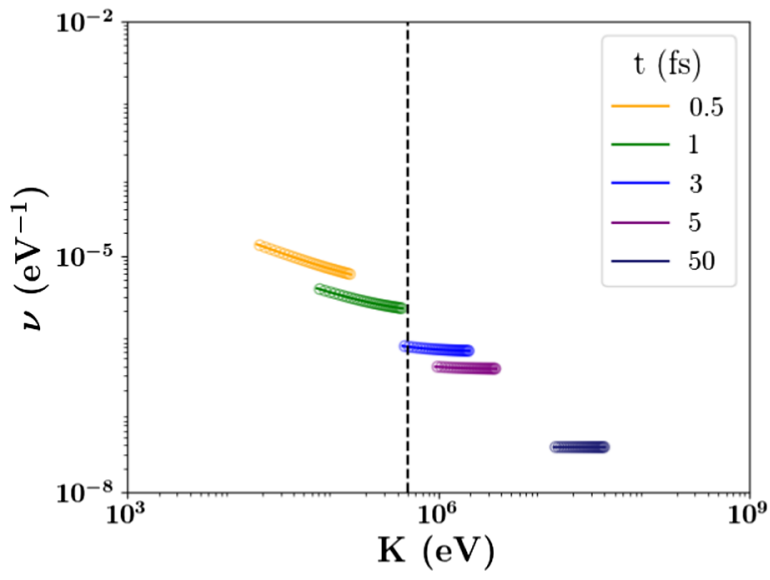

(c) $E_{a}=2 E_{T 1}$

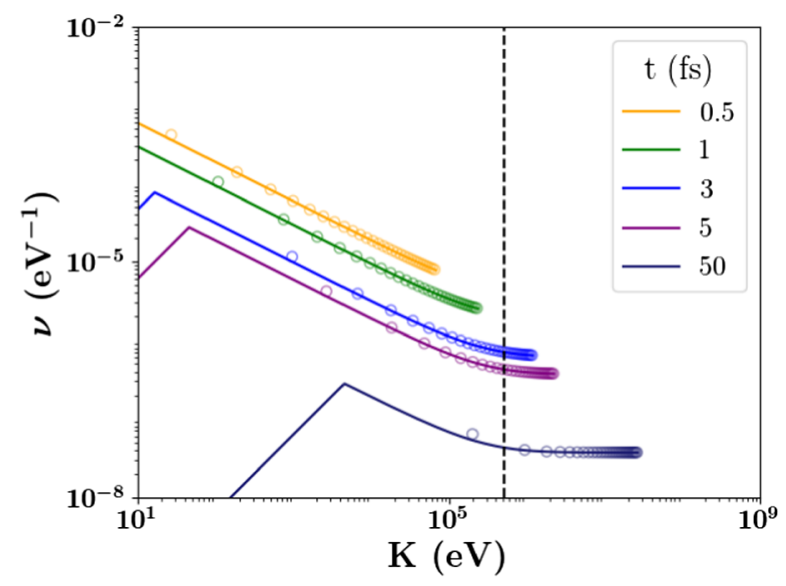

(b) $E_{a}=E_{T 1}$

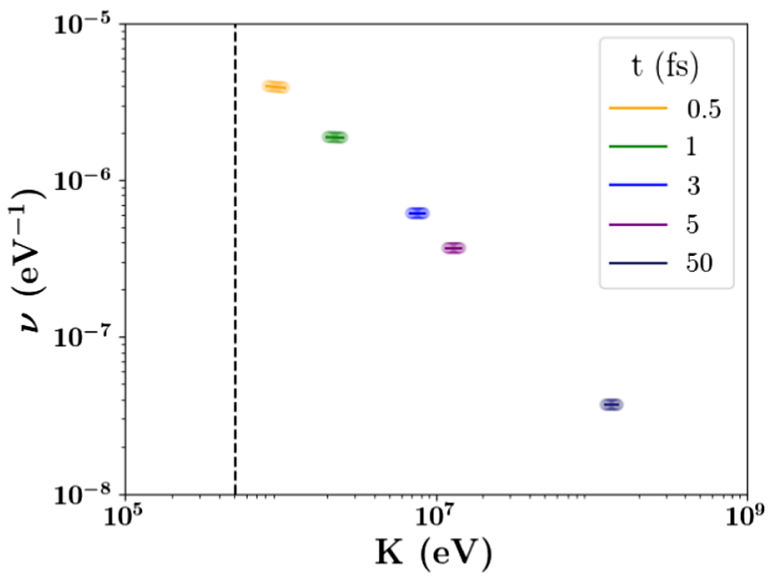

(d) $E_{a}=10 E_{T 1}$

FIG. 13. Theoretical predictions (solid line) and $M$-shell simulations (hollow dots) of the planar symmetric energy density at 4 different times with different applied extraction fields: (a) $E_{a}=0.5 E_{T 1}$, (b) $E_{a}=E_{T 1}$, (c) $E_{a}=2 E_{T 1}$, and $E_{a}=10 E_{T 1}$ where $E_{T 1}=\frac{Q_{\text {tot, } 1}}{2 \epsilon_{0}}$ and $Q_{\mathrm{tot}, 1}$ is the total surface-charge density. The results are independent of the initial profile and were obtained both from analyzing the simulations described in Figs. 11 and 12 . Notice the $\log$-log plot and the discreet jump in density seen when $E_{a}=0.5 E_{T 1}$ in (a), which coincidentally overlaps some of the $t=0.5 \mathrm{fs}$ and $t=1 \mathrm{fs}$ distributions. The energy where this jump occurs corresponds to the maximum energy of the particles moving in the $-z$-direction. At and above $E_{a}=1 E_{T 1}$ the jump is missing as the since there are no particles moving in the $-z$-direction. Also note that the energy distribution width appears to be constant once it is in the relativistic regime - this is an optical illusion of the logarithmic scale.

The asymptotic densities for the initially uniform and Gaussian distributions and for various extraction fields can be seen in Fig. 10 where we have used Eq. (39). Specifically, every point besides the point corresponding to $P_{01}+\frac{E_{a}}{E_{T 1}}=0$ will eventually have $\left|P_{01}+\frac{E_{a}}{E_{T 1}}\right| \frac{c t}{l_{r 01}} \gg 1$ and thus the density corresponding to such points will eventually become a constant. However, while $\left|P_{01}+\frac{E_{a}}{E_{T 1}}\right| \frac{c t}{l_{r 01}}$ is not much larger than 1 , the density of the point will decrease toward the eventual constant value.

On the other hand, the main effect of the extraction field on the energy distribution is that it essentially halves the distribution once the field is beyond $E_{T 1}$. This is because a freely expanding bunch has as many particles with $v$ as it does $-v$ thus doubling the number of particles with a specific kinetic energy. If $E_{a} \in\left(0, E_{T 1}\right)$, this results in the discrete jump predicted by the theory in Eq. (64).

\section{APPLICATION OF THE 1D DISTRIBUTION}

In this section, we demonstrate one use of the spatial distributions; specifically, we calculate the width evolution of UEM-relevant planar-symmetric distributions as a function of time. Specifically, we define the rms width of the distribution as

$$
\sigma_{z}=\sqrt{\left\langle z^{2}\right\rangle-\langle z\rangle^{2}}
$$




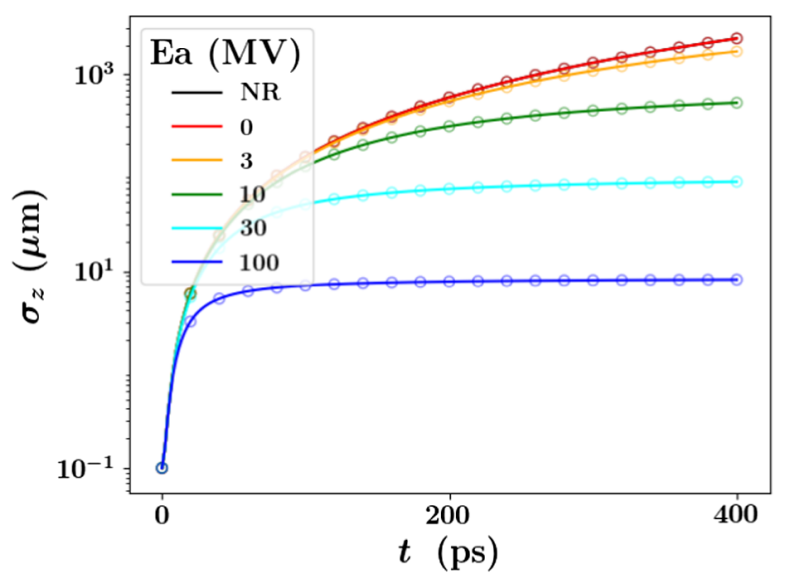

(a) $10^{6} e$

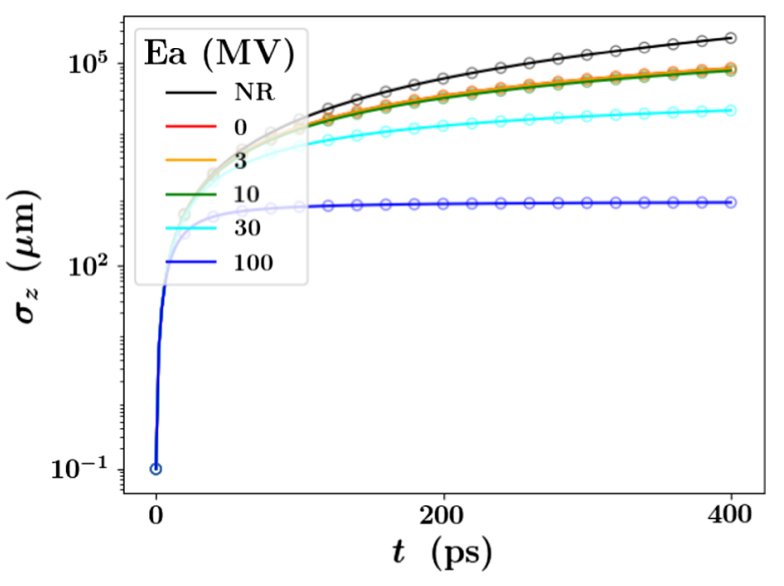

(b) $10^{8} e$

FIG. 14. Theoretical predictions (solid line) and $M$-shell simulations (hollow dots) of the width of an evolving planar-symmetric initially Gaussian distribution for two experiment relevant regimes. Both cases assumed a geometry of transverse width of $100 \mu \mathrm{m}$ and longitudinal width of $0.1 \mu \mathrm{m}$, and they differed in the number of electrons in the bunch: (a) $10^{6} e$ corresponding to an $E_{T 1} \approx 0.3 \mathrm{MV}$ and (b) $10^{8} e$ corresponding to an $E_{T 1} \approx 3 \mathrm{MV}$. Notice that the y-axis is logarithmic, and the scales are different between the two plots. Also notice that relativistic effects of spreading of the bunch in the absence of an accelerating field within this timescale are not significant for the $10^{6}$ case but noticeable for the $10^{8}$ case. Furthermore, the accelerating field effect is noticeable within this timescale when $E_{a}=10 \mathrm{MV}$ for $10^{6}$ and $E_{a}=30 \mathrm{MV}$ for $10^{8}$; however, notice that in the $10^{8}$ case, the longitudinal beam width is larger than the transverse width and that the $1 D$ model is probably no longer a valid approximation.

Theoretically $\langle a\rangle=\int_{-\infty}^{\infty} a \rho_{1} d z=\int_{-\infty}^{\infty} a\left(z_{0}\right) \rho_{01} d z_{0}$, and in simulation $\langle a\rangle=\frac{1}{N} \sum_{i=1}^{N} a_{i}$ where $N$ is the number of particles in the simulation and $a_{i}$ is the value of $a$ for the $i$ th particle.

We consider a Gaussian bunch with transverse radius of $100 \mu \mathrm{m}$ and longitudinal width of $\sigma_{r}=0.1 \mu \mathrm{m}$, and we consider both $N=10^{6}$, relevant for diffraction studies, and $N=10^{8}$, relevant for imaging studies. We treat the longitudinal expansion with the planar model both using the nonrelativistic distribution, Eq. (37), as well as the relativistic version, Eqs. (31) and (63). We calculate the theoretical expectation numerically for initially Gaussian distributed planar-symmetric distributions for various values of $E_{a}$ as well as the nonrelativistic width prediction and compare the results to the standard deviation of $M=10^{4}$-shell simulations with the same parameters. Results of this treatment may be seen in Fig. 14.

As can be seen in Fig. 14, the theory and simulations result in the same width evolution. It is worth noting that $E_{T 1} \approx 0.3 \mathrm{MV} / \mathrm{m}$ for $N=10^{6}$ and $E_{T 1} \approx 30 \mathrm{MV} / \mathrm{m}$ for $N=10^{8}$. As can be seen in the figures, the width growth does not vary much from the unaccelerated case until an extraction field is increased beyond $\approx 10 E_{T 1}$, that is the expansion dynamics will dominate the width determination until we are far beyond the "total" field within this $400 \mathrm{ps}$ time frame. Also apparent is that, within this time frame, the dynamics of the unaccelerated bunch does not differ from the nonrelativistic model; on the other hand, the higher density dynamics do differ suggesting that relativistic expansion occurs in the $N=10^{8}$ planar model.
Of course, as this bunch expands its longitudinal length will quickly become larger than the transverse width suggesting higher-dimensional dynamics will become important. Specifically after the transition to higher-dimensional dynamics, the planar model overestimates the longitudinal width and underestimates the transverse width. However, if a sufficient extraction field is obtained, i.e., around $100 E_{T 1}$, the asymptotic longitudinal width is of sufficiently small size to result in planar dynamics for the bunch meaning that the bunch width can be modeled with the planar model in that regime.

\section{DISCUSSION}

While these results compare surprisingly well with simulations, we do need to emphasize that these conclusions are based on analyzing the symmetric models at long times and that some of the physical assumptions inherent in the models should be violated at some point. The three assumptions for these model are (1) the temperature is small compared to the kinetic energy delivered to the particle due to Coulomb interaction, (2) the distributions remain laminar, and (3) the symmetry under consideration represents the physical situation. While these assumptions all break down to some extent at some point, we emphasize that in the simulations we have conducted that the model almost exactly matches with the PIC simulations.

Note that even as the distribution becomes more and more diffuse, if a region of the distribution had much less heat than the kinetic energy delivered to it by space-charge effects, we would expect the particles' trajectories to not be 
drastically altered from the trajectory determined by the space-charge effects alone-as long as the potential energy is quickly converted to kinetic energy. Nonetheless, there is always a portion of the center of the distribution that does not meet this assumption. In the planar and cylindrically symmetric cases where the kinetic energy is unbounded, this portion of the distribution is always shrinking; on the other hand, in the spherically symmetric case, there is a portion of the distribution that will never become spacecharge dominated as the kinetic energy transferred to the particles in this region will never overcome the energy associated with the initial temperature. In other words, in real world situations, the center of the distribution is emittance dominated regardless of the fact that farther out in the distribution the particles may be space-charge dominated.

The second assumption of laminar behavior is surprisingly robust for the spatial density evolution. Obviously, having a higher temperature should lead to issues with this assumption, but for the cases we have examined within the temperature range where space-charge dominated fluid is present, this does not seem to be much of an issue-at least early on. However, the energy density evolution is more sensitive to violations of the laminar assumption, and eventually these differences should be reflected in the spatial distributions. Nonetheless, the biggest success of the laminar fluid assumption is the planar symmetry case as the acceleration of successive sheets is monotonically increasing making laminar fluid assumption violating events impossible unless the initial velocity distribution is correctly tuned. The real issue with this assumption, though, is with the nonuniform bunches under cylindrical and spherical symmetries. As we have discussed here, crossover events that violate the laminar fluid assumption occur when $r^{\prime}=0$. The density shock that ends up forming in the evolution of a nonuniform bunch can be thought of as occurring in region(s) of substantial initial density that have $r^{\prime} \rightarrow 0$ relatively quickly. In other words, successive cylindrical or spherical shells begin to bunch up as they expand resulting in a relatively higher density in those regions. In the nonrelativistic case, these shells eventually crossover into the multiflow regime resulting in a violation of the laminar fluid assumption (although the model still predicts the density evolution fairly well even past such events as pointed out by other authors [33]).

On the other hand, we have considered relativistic effects here, and relativistic effects result in the density evolution being less likely to go into the multiflow regime. Specifically, the speed limit of light results in portions of the distribution going slower than if they only had nonrelativistic consideration influencing them. In the cylindrical case, the expansion may be able to "freeze" before the crossover point. On the other hand, relativity does not help the spherically symmetric case as much as complete freezing never occurs. So while $D_{02}\left(r_{0}\right)$ may adopt any negative value that can be corrected by having sufficient density, $D_{03}$ is limited by $-\frac{2}{3}$ at long times. However, even in the spherical symmetric case, the $D_{03}$ can be suppressed for arbitrary amounts of time by increasing the density. Of course for truly uniform distributions, $D_{03}=0$ everywhere and this crossover does not happen, but for any realistic distribution, all values of $D_{03}<0$ are present and crossover should occur eventually. Specifically, roughly $20 \%$ of the Gaussian distribution has $D_{03}\left(r_{0}\right)<-\frac{2}{3}$ and the crossover in this region apparently does not drastically change the evolution of the distribution as seen in the figures we presented here. As other authors have claimed that the density evolution theory captures the coulomb explosion dynamics if the multi-flow regime is small [33], we would add that relativistic effects make this agreement even better.

In the UEM community, the planar symmetric model is applied to a bunch that is thin along one axis with much larger widths along the other dimensions; we denote this as $L_{0} \ll R_{0}$ where $L_{0}$ represents the initial width of the thin dimension and $R_{0}$ the initial widths of the other two (equivalent) dimensions. If planar symmetric dynamics are present, at some time $L \approx R$, and the planar symmetric model should no longer apply instead requiring a higher dimensional description. The timescale for the expansion of the bunch is $\tau_{\exp } \approx \frac{1}{\omega_{01}}$; on the other hand, the timescale described by Eq. (68) indicates the time at which we would expect the edges of the distribution to have energy equivalent to the rest energy of the particle. As we assume $L_{0} \ll R_{0}$, we would expect that if these two timescales are of the same order or the relativistic timescale is shorter then we would expect relativistic effects described by the models presented here to occur. This occurs when $l_{r 01} \leq L_{0}$. Likewise, for the cylindrical case in fields like accelerator physics, it is generally assumed $R_{0} \ll L_{0}$; which again breaks down when $L \approx R$. Nonetheless, we again expect the cylindrically symmetric dynamics described here to be apparent if $l_{r 02} \leq R_{0}$.

Notice that in previous treatments of the evolving density $[25,32]$, the extraction field was left out of the analysis. This is accurate as the density evolution in the nonrelativistic limit, Eq. (37) is independent of the effective field. However, this is not true in the general case as relativistic effects make the electric field couple to the dynamics. This leads to an interesting opportunity to control the density through this coupling effect. Specifically, in 1D, the density freezing leads to the concept of asymptotic density, which is a density that no longer evolves in time. We showed that this asymptotic density can be manipulated through the inclusion of an extraction field, $E_{a}$. Specifically, the initial density is essentially the

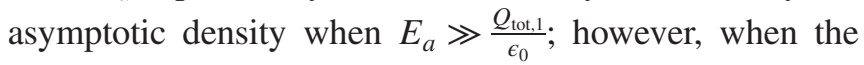
extraction field is not sufficiently large, the asymptotic density can be significant different from the initial density. 
This suggests that if we are accelerating a bunch well into the relativistic regime, we may need to consider this asymptotic density when determining optimal criteria. Namely, in the relativistic regime, an initially uniform distribution should no longer be the distribution with the smallest emittance as relativistic considerations introduce nonlinearities in the phase space that may be absent from correctly chosen initial distributions. We will develop this idea further in future work.

Recently, Kochikov et al. published an investigation of an expansion dynamics of a relativistic bunch in drift space [37]; this work uses a more sophisticated potential than what has been considered here and therefore captures additional relativistic effects. Furthermore, it extends Siwick et al.'s [23] model of bunch dynamics, that treats the width of the bunch by tracking the position of a Lagrangian particle on the symmetric axis at the fore and aft of a cylindrically symmetric bunch, into the relativistic regime. This model approximates full threedimensional effects and therefore should better model the change in the bunch width than the planar case; however, we point out that neither model accounts for the expansion of the bunch due to effective pressure introduced by emittance. We further discuss this effect in future work. Regardless, as the drift model does not treat acceleration, our planar model with acceleration is a natural complement to this theory. Furthermore, both models reach similar conclusions about expansion in the highly relativistic regime; namely that the effective reduction of the Coulomb force for a relativistic bunch suppresses the expansion of the bunch. However, we emphasize that this suppression is density dependent as the forces themselves are density dependent. Specifically, a denser bunch requires a larger velocity before the width evolution becomes slow.

\section{CONCLUSIONS}

In this work, we extended our previous density evolution analysis into the relativistic regime. Specifically, we showed that the uniform distribution in any dimension develops density shocks as the outer portion of the distribution becomes relativistic; we also found expressions for such peaks in other distributions which occur in competition with the nonuniform Coulomb mechanism that leads to peaks in such distributions [32]. We showed that the analytic results accurately predicted 1D-like $M$-shell simulation results under all symmetries and PIC results under cylindrical and spherical symmetries. The PIC simulations conducted here were completed using an EM solver with an initial ES solve used to initialize the fields. As these simulations agree with the theory that is essentially based on electrostatics, it is apparent that EM effects beyond electrostatics are not significantly affecting the spatial density evolution and the energy density evolution for the problems examined.
We emphasize that the mechanism for the relativistic shock development is distinct from nonrelativistic shock development seen in the Gaussian distribution [32]. Previously, we demonstrated that shocks arise in nonplanar nonuniform spatial distribution evolutions due to the initial distribution leading to nonlinear Lagrangian particle velocities that lead to inner Lagrangian particles catching up to outer Lagrangian particles. On the other hand, shock in a relativistic bunch is caused by the "shrinking" of onedimension of the density as the Lagrangian particles approach the luminal speed limit. This can be seen by considering the energy of continuum particles within the distribution. Specifically, as the particles expand, their kinetic energies increase according to Eq. (9). In the planar and cylindrically symmetric models, this increase is linear and logarithmic in their position (and eventually time), respectively, as can be seen by Eqs. (10) and (11). This leads to all particles in a neighborhood approaching the speed of light resulting in the "freezing" of the expansion along the expanding dimension; that is, all planar symmetric distributions eventually asymptote to a time independent density while all cylindrically symmetric distributions eventually expand "uniformlike" but with one dimension less than that being considered, i.e., $\rho_{2} \rightarrow$ $\frac{r_{0}}{r} A \rho_{02}$ where $A$ is some parameter determined from the initial conditions. On the other hand, in the spherically symmetric case, the kinetic energy is bounded by $\frac{q Q_{\text {tot } 3} P_{03}}{4 \pi \epsilon_{0} r_{0}}$, which is finite. As this kinetic energy is dependent on $r_{0}$ through $\frac{P_{03}\left(r_{0}\right)}{r_{0}}$, the asymptotic velocity of neighboring continuum particles differs. This is why the density at long times for highly relativistic portions of the distribution drops in a uniformlike manner, i.e., $\rho_{3} \rightarrow \frac{r_{0}^{3}}{r^{3}} A \rho_{03}$ with $A=\frac{1+3 \zeta^{2}+2 \zeta^{4}}{1+\frac{3}{2} D_{03}}$ [see Eq. (59)]. While both the planar and cylindrically symmetric cases lost a power to the uniformlike evolution, i.e., planar cases evolve like $\frac{1}{r^{0}}$ and cylindrically symmetric cases evolve like $\frac{1}{r^{1}}$, the spherically symmetric case's uniformlike evolution retains $\frac{1}{r^{3}}$. This difference arises as the particles in the spherically symmetric case have finite potential energy and thus asymptote toward a velocity that is a little less than the speed of light. Now neighboring Lagrangian particles can have very small differences in their asymptotic velocity leading to the expansion in the radial direction being slower than what is seen nonrelativistically; specifically, this is what leads to $A>1$. However, there does remain a nonvanishing small velocity difference meaning that the distribution continues to expand in all dimensions. Nonetheless, these effects lead to density peaks forming toward the edges of the distribution in all cases; regardless, we find it interesting that the behavior in each dimension is qualitatively unique.

We also added an extraction field to the laminar, planar theory, and we rederived a threshold field, $E_{T 1}=\frac{Q_{\text {tot.1 }}}{2 \epsilon_{0}}$, that 
is responsible for qualitatively different behavior in the evolution of the density. This threshold is the virtual cathode limit identified previously by Valfells [36] and generally discussed in the accelerator literature. For $10^{8}$ electrons in a uniform bunch of radius $100 \mu \mathrm{m}, E_{T 1} \approx$ $30 \mathrm{MV} / \mathrm{m}$; thus an acceleration field of $100 \mathrm{MV}$, which is the upper limit of the UEM community used at the Stanford Linear Accelerator [38-40], is only about $3.5 \times$ this quantity. As we saw in Figs. (11) and (12), in this range we would still expect space-charge effects that enact substantial expansion and distortion of the initial distribution. On the other hand, table top UEM devices typically have extraction fields up to $5 \mathrm{MV} / \mathrm{m}$ [41-44], which is only slightly more than the total internal field of $10^{7}$ electrons in a pancake with a radius of $100 \mu \mathrm{m}, E_{T 1} \approx$ $3 \mathrm{MV} / \mathrm{m}$ and is far below $E_{T 1}$ for $10^{8}$ electrons. Thus $10^{8}$ electron bunches are beyond the capability of such table top devices, and $10^{7}$ electron bunches should expand immensely within the extraction field making them very difficult to work with.

A common effect in all dimensions, was that if the distribution is given enough time to expand, the stochastic effects in the initial distribution are overwhelmed by the space charge effects. This means that under such conditions, repeated instances of similar bunches should look more or less the same. However, if the bunch is quickly accelerated into the relativistic regime, the initial stochastic fluctuations are preserved in the density profile. As we move to larger accelerating fields, this effect needs to be further studied as it may be that stochastics will play a larger role in the limit of very large extraction fields.

\section{ACKNOWLEDGMENTS}

This work was supported by NSF Grants No. 1625181 and No. 1803719 and the College of Natural Science at Michigan State University. Computational resources were provided by the High Performance Computer Center at MSU.

\section{APPENDIX A: CYLINDRICAL SYMMETRIC DENSITY EVOLUTION IN THE HIGHLY RELATIVISTIC REGIME}

Assuming $2 \tilde{\beta}^{2} \gg 1$ and analyzing all but $\mathcal{F}$ and $\mathcal{F}_{\partial}$, Eq. (48) becomes

$$
r^{\prime} \approx \frac{r}{r_{0}}\left[1+\left(\frac{\rho_{0}}{\bar{\rho}_{0}}-1\right) \frac{1}{\tilde{\beta}} \mathcal{F}-\frac{\rho_{0}}{\bar{\rho}_{0}} \mathcal{F}_{\partial}\right]
$$

However,

$$
\mathcal{F} \approx \frac{r_{0}}{r} \int_{0}^{\sqrt{\ln \left(\frac{r}{r_{0}}\right)}} 2 \tilde{\beta} y e^{y^{2}} d y=\tilde{\beta}\left(1-\frac{r_{0}}{r}\right)
$$

and

$$
\mathcal{F}_{\partial} \approx \frac{r_{0}}{r} \int_{0}^{\sqrt{\ln \left(\frac{r}{r_{0}}\right)}} 2 y e^{y^{2}} d y=1-\frac{r_{0}}{r}
$$

Placing these approximations back into Eq. (C1) results in $r^{\prime} \approx 1$.

\section{APPENDIX B: LONG-TIME LIMIT}

Consider spherical symmetry. Notice that $\frac{r_{0}}{r} r^{\prime}$ is a function of $\frac{r_{0}}{r}$, so in the limit $\lim _{\frac{r}{r_{0}} \rightarrow \infty}$, these terms go to zero. As a result, $x \rightarrow 1$ in the expression for $r^{\prime}$, so

$$
\begin{aligned}
\lim _{r_{r_{0}} \rightarrow \infty} r^{\prime}= & \frac{r}{r_{0}}\left[1+\frac{p_{1}(1)}{b_{1}^{2}\left[g_{2}(1)\right]^{2}}\right. \\
& \left.+\frac{r_{0}}{r} \frac{p_{2}(1)}{b_{1}^{2}\left[g_{2}(1)\right]^{2}} T\left(\sqrt{1-\frac{r_{0}}{r}}\right)\right] \\
= & \frac{r}{r_{0}}\left[\frac{\left(1+\frac{3}{2} D\right)\left(1+\tilde{\beta}^{2}\right)}{\left(1+\tilde{\beta}^{2}\right)^{2}\left(1+2 \tilde{\beta}^{2}\right)}\right] \\
& +\frac{\left(3 \tilde{\beta}^{2}+6 \tilde{\beta}^{2} D+\frac{3}{2} D\right) \tanh ^{-1} \sqrt{1-\frac{r_{0}}{r}}}{\left(1+\tilde{\beta}^{2}\right)^{2}\left(1+2 \tilde{\beta}^{2}\right)} \\
& \rightarrow \frac{r}{r_{0}}\left(\frac{1+\frac{3}{2} D}{1+3 \tilde{\beta}^{2}+2 \tilde{\beta}^{4}}\right)
\end{aligned}
$$

where $\tilde{\beta}=\frac{r_{0} \bar{\omega}_{03}}{\sqrt{6} c}$ and where the second term is lost since inverse hyperbolic tangent goes to infinity logarithmically which is slower than $\frac{r}{r_{0}}$.

\section{APPENDIX C: HIGH DENSITY LIMIT}

At high densities, the edges of the planar symmetric distribution do not significantly evolve, and therefore the distribution is essentially preserved in this region. This occurs when $\frac{2 \omega_{01} L_{0}}{c} \gg 1$. We now extend this to the other symmetries.

\section{Cylindrical symmetry}

Assuming $\tilde{\beta} \gg 1$, where $\tilde{\beta}=\frac{r_{0} \bar{\omega}_{02}}{2 c}$, and analyzing all but $\mathcal{F}$ and $\mathcal{F}_{\partial}$, Eq. (r') becomes

$$
r^{\prime} \approx \frac{r}{r_{0}}\left(1+\left(\frac{\rho_{0}}{\bar{\rho}_{0}}-1\right) \frac{1}{\tilde{\beta}} \mathcal{F}-\frac{\rho_{0}}{\bar{\rho}_{0}} \mathcal{F}_{\partial}\right)
$$

However,

$$
\mathcal{F} \approx \frac{r_{0}}{r} \int_{0}^{\sqrt{\ln \left(\frac{r}{r_{0}}\right)}} 2 \tilde{\beta} y e^{y^{2}} d y=\tilde{\beta}\left(1-\frac{r_{0}}{r}\right)
$$

and

$$
\mathcal{F}_{\partial} \approx \frac{r_{0}}{r} \int_{0}^{\sqrt{\ln \left(\frac{r}{r_{0}}\right)}} 2 y e^{y^{2}} d y=1-\frac{r_{0}}{r}
$$


Placing these approximations back into Eq. (C1) results in $r^{\prime} \approx 1$. That is, $r^{\prime}$ cancels out the factor $\frac{r}{r_{0}}$ term.

\section{Spherical symmetry}

Assuming $\tilde{\beta} \gg 1$, where $\tilde{\beta}=\frac{r_{0} \bar{\omega}_{03}}{\sqrt{6} c}$, we see that Eq. (B1) is approximately 0 ; therefore we need to return to the full expression and expand in terms of $\frac{r}{r_{0}}$. We find only keeping the highest order of $\tilde{\beta}$

$$
\begin{aligned}
r^{\prime} & \approx \frac{r}{r_{0}}\left[1+\frac{-2 \tilde{\beta}^{2}\left(1-\frac{r_{0}}{r}\right)^{2}}{2 \tilde{\beta}^{6}\left(1-\frac{r_{0}}{r}\right)}\right] \\
& =\frac{r}{r_{0}}\left[1-\left(1-\frac{r_{0}}{r}\right)\right] \\
& =1
\end{aligned}
$$

So like the planar and cylindrical symmetric cases, superhighly relativistic densities result in essentially the loss of one dimension during expansion.

[1] G. H. Jansen, Coulomb Interactions in Particle Beams (Technische Universiteit Delft, Delft, 1988).

[2] M. Reiser, Theory and Design of Charged Particle Beams (John Wiley \& Sons, New York, 1994).

[3] Y. K. Batygin, Self-consistent analysis of three-dimensional uniformly charged ellipsoid with zero emittance, Phys. Plasmas 8, 3103 (2001).

[4] V. Y. Bychenkov and V. Kovalev, Coulomb explosion in a cluster plasma, Plasma Phys. Rep. 31, 178 (2005).

[5] M. Grech, R. Nuter, A. Mikaberidze, P. Di Cintio, L. Gremillet, E. Lefebvre, U. Saalmann, J. M. Rost, and S. Skupin, Coulomb explosion of uniformly charged spheroids, Phys. Rev. E 84, 056404 (2011).

[6] A. E. Kaplan, B. Y. Dubetsky, and P. L. Shkolnikov, Shock Shells in Coulomb Explosions of Nanoclusters, Phys. Rev. Lett. 91, 143401 (2003).

[7] V. Kovalev and V. Y. Bychenkov, Kinetic description of the Coulomb explosion of a spherically symmetric cluster, J. Exp. Theor. Phys. 101, 212 (2005).

[8] I. Last, I. Schek, and J. Jortner, Energetics and dynamics of Coulomb explosion of highly charged clusters, J. Chem. Phys. 107, 6685 (1997).

[9] M. Eloy, R. Azambuja, J. Mendonca, and R. Bingham, Interaction of ultrashort high-intensity laser pulses with atomic clusters, Phys. Plasmas 8, 1084 (2001).

[10] V.P. Krainov and A. S. Roshchupkin, Dynamics of the Coulomb explosion of large hydrogen iodide clusters irradiated by superintense ultrashort laser pulses, Phys. Rev. A 64, 063204 (2001).

[11] J. P. Morrison and E. R. Grant, Dynamics of colliding ultracold plasmas, Phys. Rev. A 91, 023423 (2015).

[12] E. Boella, B. P. Paradisi, A. DAngola, L. O. Silva, and G. Coppa, Study on Coulomb explosions of ion mixtures, J. Plasma Phys. 82, 905820110 (2016).
[13] V. Y. Bychenkov and V. F. Kovalev, Relativistic coulomb explosion of spherical microplasma, JETP Lett. 94, 97 (2011).

[14] O. Anderson, Internal dynamics and emittance growth in space charge dominated beams, Part. Accel. 21, 197 (1987).

[15] J. Rosenzweig, A. Cook, R. England, M. Dunning, S. Anderson, and M. Ferrario, Emittance compensation with dynamically optimized photoelectron beam profiles, Nucl. Instrum. Methods Phys. Res., Sect. A 557, 87 (2006).

[16] R. L. Gluckstern, Analytic Model for Halo Formation in High Current Ion Linacs, Phys. Rev. Lett. 73, 1247 (1994).

[17] T. P. Wangler, Emittance growth from space-charge forces, Los Alamos National Lab. Technical Report, 1991.

[18] V. P. Degtyareva, M. A. Monastyrsky, M. Y. Schelev, and V. A. Tarasov, Computer studies on dynamics of ultrashort electron packets in streak tubes and diffractometers, Opt. Eng. 37, 2227 (1998).

[19] O. J. Luiten, S. B. vanderGeer, M. J. deLoos, F. B. Kiewiet, and M. J. vanderWiel, How to Realize Uniform ThreeDimensional Ellipsoidal Electron Bunches, Phys. Rev. Lett. 93, 094802 (2004).

[20] P. Musumeci, J. T. Moody, R. J. England, J. B. Rosenzweig, and T. Tran, Experimental Generation and Characterization of Uniformly Filled Ellipsoidal Electron-Beam Distributions, Phys. Rev. Lett. 100, 244801 (2008).

[21] V. R. Morrison, R. P. Chatelain, C. Godbout, and B. J. Siwick, Direct optical measurements of the evolving spatio-temporal charge density in ultrashort electron pulses, Opt. Express 21, 21 (2013).

[22] Y. Li and J. W. Lewellen, Generating a Quasiellipsoidal Electron Beam by 3D Laser-Pulse Shaping, Phys. Rev. Lett. 100, 074801 (2008).

[23] B. J. Siwick, J. R. Dwyer, R. E. Jordan, and R. J. Dwayne Miller, Ultrafast electron optics: Propagation dynamics of femtosecond electron packets, J. Appl. Phys. 92, 1643 (2002).

[24] B.-L. Qian and H. E. Elsayed-Ali, Electron pulse broadening due to space charge effects in a photoelectron gun for electron diffraction and streak camera systems, J. Appl. Phys. 91, 462 (2002).

[25] B. W. Reed, Femtosecond electron pulse propagation for ultrafast electron diffraction, J. Appl. Phys. 100, 034916 (2006).

[26] S. Collin, M. Merano, M. Gatri, S. Sonderegger, P. Renucci, J.-D. Ganiere, and B. Deveaud, Transverse and longitudinal space-charge-induced broadenings of ultrafast electron packets, J. Appl. Phys. 98, 094910 (2005).

[27] A. Gahlmann, S. T. Park, and A. H. Zewail, Ultrashort electron pulses for diffraction, crystallography and microscopy: Theoretical and experimental resolutions, Phys. Chem. Chem. Phys. 10, 2894 (2008).

[28] Z. Tao, H. Zhang, P. Duxbury, M. Berz, and C.-Y. Ruan, Space charge effects in ultrafast electron diffraction and imaging, J. Appl. Phys. 111, 044316 (2012).

[29] J. Portman, H. Zhang, Z. Tao, K. Makino, M. Berz, P. Duxbury, and C.-Y. Ruan, Computational and experimental characterization of high-brightness beams for 
femtosecond electron imaging and spectroscopy, Appl. Phys. Lett. 103, 253115 (2013).

[30] J. Portman, H. Zhang, K. Makino, C. Ruan, M. Berz, and P. Duxbury, Untangling the contributions of image charge and laser profile for optimal photoemission of high-brightness electron beams, J. Appl. Phys. 116, 174302 (2014).

[31] A. Michalik and J. Sipe, Analytic model of electron pulse propagation in ultrafast electron diffraction experiments, J. Appl. Phys. 99, 054908 (2006).

[32] B. Zerbe, X. Xiang, C.-Y. Ruan, S. Lund, and P. Duxbury, Dynamical bunching and density peaks in expanding Coulomb clouds, Phys. Rev. Accel. Beams 21, 064201 (2018).

[33] V. Kovalev, K. Popov, V. Y. Bychenkov, and W. Rozmus, Laser triggered Coulomb explosion of nanoscale symmetric targets, Phys. Plasmas 14, 053103 (2007).

[34] D. Murphy, R. Speirs, D. Sheludko, C. Putkunz, A. McCulloch, B. Sparkes, and R. Scholten, Detailed observation of spacecharge dynamics using ultracold ion bunches, Nat. Commun. 5, 4489 (2014).

[35] A. Friedman, R. H. Cohen, D. P. Grote, S. M. Lund, W. M. Sharp, J.-L. Vay, I. Haber, and R. A. Kishek, Computational Methods in the Warp Code Framework for Kinetic Simulations of Particle Beams and Plasmas, IEEE Trans. Plasma Sci. 42, 1321 (2014).

[36] A. Valfells, D. Feldman, M. Virgo, P. O'shea, and Y. Lau, Effects of pulse-length and emitter area on virtual cathode formation in electron guns, Phys. Plasmas 9, 2377 (2002).
[37] I. Kochikov, R. D. Miller, and A. Ischenko, Relativistic Modeling of Ultra-Short Electron Pulse Propagation, J. Exp. Theor. Phys. 128, 333 (2019).

[38] P. Musumeci, J. Moody, C. Scoby, M. Gutierrez, M. Westfall, and R. Li, Capturing ultrafast structural evolutions with a single pulse of $\mathrm{MeV}$ electrons: Radio frequency streak camera based electron diffraction, J. Appl. Phys. 108, 114513 (2010).

[39] S. Weathersby et al., Mega-electron-volt ultrafast electron diffraction at SLAC National Accelerator Laboratory, Rev. Sci. Instrum. 86, 073702 (2015).

[40] Y. Murooka, N. Naruse, S. Sakakihara, M. Ishimaru, J. Yang, and K. Tanimura, Transmission-electron diffraction by $\mathrm{MeV}$ electron pulses, Appl. Phys. Lett. 98, 251903 (2011).

[41] R. Srinivasan, V. Lobastov, C.-Y. Ruan, and A. Zewail, Ultrafast electron diffraction (UED), Helv. Chim. Acta 86, 1761 (2003).

[42] C.-Y. Ruan, Y. Murooka, R. K. Raman, R. A. Murdick, R. J. Worhatch, and A. Pell, The development and applications of ultrafast electron nanocrystallography, Microsc. Microanal. 15, 323 (2009).

[43] T. vanOudheusden, P. L. E. M. Pasmans, S. B. vanderGeer, M. J. deLoos, M. J. vanderWiel, and O. J. Luiten, Compression of Subrelativistic Space-Charge-Dominated Electron Bunches for Single-Shot Femtosecond Electron Diffraction, Phys. Rev. Lett. 105, 264801 (2010).

[44] G. Sciaini and R. D. Miller, Femtosecond electron diffraction: Heralding the era of atomically resolved dynamics, Rep. Prog. Phys. 74, 096101 (2011). 